\title{
Streptococcus suis: a re-emerging pathogen associated with occupational exposure to pigs or pork products. Part II - Pathogenesis
}

\author{
Jacek Dutkiewicz ${ }^{1, A-F}$, Violetta Zając ${ }^{1, C-D}$, Jacek Sroka' ${ }^{1,2, B, F}$, Bernard Wasiński ${ }^{3, E-F}$, Ewa Cisak ${ }^{1, E-F}$, \\ Anna Sawczyn ${ }^{1, D-F}$, Anna Kloc ${ }^{1, D-E}$, Angelina Wójcik-Fatla ${ }^{1, A-F}$ \\ ${ }^{1}$ Department of Health Biohazards and Parasitology, Institute of Rural Health, Lublin, Poland \\ 2 Department of Parasitology, National Veterinary Research Institute, Puławy, Poland \\ ${ }^{3}$ Department of Hygiene of Food of Animal Origin, National Veterinary Research Institute, Puławy, Poland \\ A - Research concept and design, B - Collection and/or assembly of data, C - Data analysis and interpretation, \\ $D$ - Writing the article, $E$ - Critical revision of the article, $F$ - Final approval of article
}

Dutkiewicz J, Zając V, Sroka J, Wasiński B, Cisak E, Sawczyn A, Kloc A, Wójcik-Fatla A. Streptococcus suis: a re-emerging pathogen associated with occupational exposure to pigs or pork products. Part II - Pathogenesis. Ann Agric Environ Med. 2018; 25(1): 186-203. doi: 10.26444/aaem/85651

\section{Abstract}

Streptococcus suis is a re-emerging zoonotic pathogen that may cause severe disease, mostly meningitis, in pigs and in humans having occupational contact with pigs and pork, such as farmers, slaughterhose workers and butchers. The first stage of the pathogenic process, similar in pigs and humans, is adherence to and colonisation of mucosal and/or epithelial surface(s) of the host. The second stage is invasion into deeper tissue and extracellular translocation of bacterium in the bloodstream, either free in circulation or attached to the surface of monocytes. If $S$. suis present in blood fails to cause fatal septicaemia, it is able to progress into the third stage comprising penetration into host's organs, mostly by crossing the blood-brain barrier and/or blood-cerebrospinal fluid barrier to gain access to the central nervous system (CNS) and cause meningitis. The fourth stage is inflammation that plays a key role in the pathogenesis of both systemic and CNS infections caused by S. suis. The pathogen may induce the overproduction of pro-inflammatory cytokines that cause septic shock and/or the recruitment and activation of different leukocyte populations, causing acute inflammation of the CNS. Streptococcus suis can also evoke - through activation of microglial cells, astrocytes and possibly other cell types - a fulminant inflammatory reaction of the brain which leads to intracranial complications, including brain oedema, increased intracranial pressure, cerebrovascular insults, and deafness, as a result of cochlear sepsis.

In all stages of the pathogenic process, S. suis interacts with many types of immunocompetent host's cells, such as polymorphonuclear leukocytes, mononuclear macrophages, lymphocytes, dendritic cells and microglia, using a range of versatile virulence factors for evasion of the innate and adaptive immune defence of the host, and for overcoming environmental stress. It is estimated that $S$. suis produces more than 100 different virulence factors that could be classified into 4 groups: surface components or secreted elements, enzymes, transcription factors or regulatory systems and transporter factors or secretion systems. A major virulence factor is capsular polysaccharide (CPS) that protects bacteria from phagocytosis. However, it hampers adhesion to and invasion of host's cells, release of inflammatory cytokines and formation of the resistant biofilm which, in many cases, is vital for the persistence of bacteria. It has been demonstrated that the arising by mutation unencapsulated $S$. suis clones, which are more successful in penetration to and propagation within the host's cells, may coexist in the organism of a single host together with those that are encapsulated. Both 'complementary' clones assist each other in the successful colonization of host's tissues and persistence therein.

S. suis has an open pan-genome characterized by a frequent gene transfer and a large diversity. Of the genetic determinants of S. suis pathogenicity, the most important are pathogenicity islands (PAI), in particular, a novel DNA segment of $89 \mathrm{~kb}$ length with evident pathogenic traits that has been designated as $89 \mathrm{~K} \mathrm{PAI}$. It has been estimated that more than one-third of the $S$. suis virulence factors is associated with this PAl. It has been proved that the virulent $S$. suis strains possess smaller genomes, compared to avirulent ones, but more genes associated with virulence. Overall, the evolution of the species most probably aims towards increased pathogenicity, and hence the most significant task of the current research is an elaboration of a vaccine, efficient both for humans and pigs.

\section{Key words}

Streptococcus suis, pathogenesis, infection stages, interaction with host cells and tissues, virulence factors, genomic determinants of pathogenicity, biofilm formation

\section{INTRODUCTION}

Streptococcus suis, a commensal of upper respiratory tract of pigs, can cause serotype (type)-dependent disease both in

Address for correspondence: Jacek Dutkiewicz, Department of Health Biohazards and Parasitology, Institute of Rural Health, Lublin, Poland, Jaczewskiego 2, 20-090 Lublin, Poland

e-mail: jadut777@onet.eu

Received: 29.01.2018; accepted: 22.02.2018; first published:02.03.2018 pigs and humans, mostly manifested as meningitis [1-4]. In the epidemics that occurred in 2005 in the Chinese province of Sichuan, a marked part of patients infected with S. suis experienced a shock-like syndrome with a high mortality, that has been described in the preceding article devoted to epidemiology of the disease caused by this bacterium [5]. Since then, a number of studies on pathogenesis of porcine streptococcosis grew exponentially and supported important information on the stages of pathogenic process and virulence 
factors, which are reviewed below. Nevertheless, a number of gaps persist that hopefully which be filled in the not too distant future.

\section{PATHOGENESIS}

Stages of the pathogenic process. Based on the source articles by Gottschalk et al. [1], Fittipaldi et al. [2], Fulde \& Valentin-Wiegand [3], Feng et al. [4], Segura et al. [6], Kouki et al. [7] and Auger \& Gottschalk [8], the following stages of the pathogenic process in swine and/or humans can be distinguished:

- adherence to and colonisation of mucosal and/or epithelial surface(s) of the host;

- invasion into deeper tissue and translocation in the bloodstream;

- breaching of the blood-brain barrier;

- inflammation.

Adherence to and colonisation of mucosal and/or epithelial surface(s) of the host. Pigs become infected with S. suis mostly by the airborne route 'nose-to-nose'; the palatine and pharyngeal tonsils are potential portals of entry for this pathogen. By contrast, the main route of entry of the organism in humans proceeds through small injuries causing a breach of the skin integrity. While the airborne route is less important in humans, a part of infections, mostly in Asia, occur by the gastrointestinal route, through consumption of raw pig meat or blood $[1,6]$.

So far, little is known about the initial steps of the pathogenesis of infection caused by $S$. suis, including mucosal colonization and the host innate immune response. In these steps, the primary role is played by adhesins produced by the pathogen. In a study on the initial stages of $S$. suis infection, Segura et al. [6] list 33 specific S. suis proteins or other adhesins enabling adhesion to host mucosal epithelial cells, including known virulence factors: suilysin, surface antigen one (Sao), fibronectin-binding protein (Ssa), autolysin (atl) and various enzymes. These authors also list 13 regulatory genes reported to modulate adhesion to epithelial cells, such as CodY or serine/threonine phosphatase (STP). Mucosal colonization and subsequent invasion of the submucosal connective tissue by $S$. suis is facilitated by the ability to bind to the extracellular matrix (ECM) components (such as fibrinogen/fibrin, fibronectin, laminin, different types of collagen, vitronectin, and/or proteoglycans), and by plasminogen activation which results in the convertion of plasminogen to plasmin protease that causes fibrin degradation and tissue destruction. The bacterial molecules involved in such interactions include enolase, muramidase-released protein (MRP), fibronectinbinding protein (Ssa), and autolysin (atl) [6].

The role of capsular polysaccharide (CPS), one of the most important virulence factors, on bacterial adhesion to epithelial cells and invading these cells is controversial. On the one hand, CPS interferes with adhesion/invasion of epithelial cells (by adhesin hindering and biophysical repulsion), and therefore during colonisation of the mucosal epithelium by S. suis the capsule is probably downregulated to allow adherence and invasion of the bacterium to overcome this first barrier. On the other hand, the capsule promotes penetration of the pathogen through mucus layer to the epithelial surface $[3,6,7]$.
In early infection, an important role is played by enzymes produced by S. suis, mostly proteases (such as fibrinogendegrading subtilisin) and deoxyribonucleases (DNases). They are involved in bacterial growth, biofilm formation and evasion of the host immune system. The enzymes were detected in $S$. suis membrane vesicles, small globular structures of 10-200 $\mathrm{nm}$ diameter formed by membrane blebbing, which can promote colonization by reaching areas not accessible to the whole bacteria [6].

Little is known about the mechanisms by which $S$. suis bacteria overcome the innate defence mechanism of the host. Surface-associated subtilisin-like protease (SspA), lipoteichoic acid (LTA) $D$-alanylation and peptidoglycan (PG) N-deacetylation contribute to enhanced resistance to antimicrobial polypeptides, such as lysozyme, defensins and cathelicidins, secreted by epithelial cells and/or innate immune cells including neutrophils. The expression of SspA and of another unidentified serine protease by $S$. suis, which cleave the CCL5 and CXCL8 chemokines secreted by host cells, enables the bacterium to avoid and/or delay leukocyte attraction and activation. The bacteria produce also IgA and IgM proteases targeting the host immunoglobulins, as well as adenosine synthase (Ssad), nuclease A (SsnA) and endonuclease A (EndAsuis) that promote protection against the antimicrobial effects of neutrophils or the neutrophil extracellular traps (NETs). Moreover, S. suis has developed several mechanisms to inactivate the host complement [6].

A limited number of $S$. suis carbohydrate-based adhesion mechanisms have been described. Kouki et al. [7] identified the streptococcal adhesin $\mathrm{P}(\mathrm{SadP})$, encoded by the $S$. suis gene $s a d P$, that binds to Gal $\alpha 1-4 \mathrm{Gal}$ (galabiose)-oligosaccharides present in host glycolipids. SadP has a Gal $\alpha 1-4$ Gal-binding $N$-terminal domain and a $C$-terminal LPNTG-motif for cell wall anchoring. According to the authors, SadP adhesin, recently regarded as identical with the factor H-binding protein [8], may represent a promising target for the design of anti-adhesion ligands for the prevention and treatment of $S$. suis infections.

Invasion into deeper tissue and translocation in the bloodstream. A hallmark of S. suis pathogenicity is its ability to disseminate in the blood circulation and to maintain bacteraemia for certain time. This is considered to be crucial in causing meningitis. Thus, major mechanisms in pathogenesis of $S$. suis infections are those involved in (i) invasion of S. suis through the epithelial cell barriers, (ii) evasion of killing by complement and phagocytosis, and (iii) invasion into the cerebrospinal fluid (CSF) or other target sites [3].

It is not clear how $S$. suis penetrates the first line of host defence to be disseminated by blood and/or lymph and initiate disease. Most probably, bacteria breach the mucosal epithelium in the upper respiratory tract of pigs. Similarly, in humans, $S$. suis could interact with the epidermal cells at skin wounds or with the mucosal epithelial cells of the intestine in the oral route of infection $[1,6]$. Ferrando et al. [9] provided experimental evidence indicating that the gastrointestinal tract could be an entry site of $S$. suis type 2 infection, both in pigs and humans.

At this stage, the most important functions of S. suis are those aimed at overcoming stress conditions and host immune defence. At present, little is known about the mechanisms of $S$. suis metabolic adaptation to various stress 
conditions, e.g. low $\mathrm{pH}$ or low availability of nutrients and oxygen. A recently identified S. suis catabolic enzyme system, the arginine deiminase system (ADS), may play an important role in adaptation to such conditions. This is a secondary enzymatic system catabolising arginine, and concomitantly producing citrulline, ATP, $\mathrm{CO}_{2}$ and ammonia. The system is regulated by a variety of environmental stimuli, such as $\mathrm{pH}$, carbon starvation, oxygen content and substrate availability. It enables $S$. suis to alkalize environment by synthesis of ammonia during arginine catabolism, and thereby protect itself against acidic damage in phagolysosomes [3].

Streptococcus suis is able to survive in the bloodstream and disseminate to the central nervous system (CNS) and other organs of the host, thanks to the efficient protection provided by CPS against neutrophils- and monocyte/macrophagemediated phagocytosis and killing. According to the early theory of a 'Trojan horse', bacteria are taken-up by monocytes (in the absence of specific antibodies), survive intracellularly and then invade the CNS. However, contemporary studies conducted by different laboratories demonstrate that bacteria travel extracellularly, either free in circulation or attached to the surface of monocytes [1]. Phagocytic cells are unable to efficiently phagocytose encapsulated $S$. suis in the absence of specific antibodies, and suilysin further protects bacteria upon complement-mediated uptake and killing. In conclusion, Gottschalk et al. [1] and Segura et al. [10] expressed the opinion that $S$. suis can be considered a truly extracellular systemic pathogen.

Virulent $S$. suis strains are able to maintain a high bacterial concentration in the blood. Subsequently, lipoproteins and other factors present in the bacterial cell wall trigger a fulminant release of inflammatory mediators that can lead to a toxic shock-like syndrome [2].

Crossing of the blood-brain barrier. After survival in blood and following dissemination, S. suis invades multiple organs, including the spleen, liver, kidney, lung, and heart. If $S$. suis present in blood fails to cause fatal septicaemia, it is able to cross the blood-brain barrier (BBB) and/or blood-cerebrospinal fluid barrier (BCSFB) made by the brain microvascular endothelial cells (BMECs) and/or the choroid plexus epithelial cells (CPECs) to gain access to CNS and cause meningitis. The mechanisms by which bacteria reach the CNS are only partially known and include adhesion to BMECs or CPECs, which have been reported to be independent of the capsular polysaccharide expression and mediated by cell wall components, such as the LTA, LPXTG cell wall-anchored proteins and enolase. The next step is penetration into these cells either by direct invasion of bacteria into the cells by endocytic mechanisms, or by disrupting the cell junctions. S. suis can also bind and activate plasminogen to proteolytically render the $\mathrm{BBB}$ leaky to bacteria. Furthermore, the S. suis CPS and cell wall can synergistically induce human macrophages to secrete prostaglandin $\mathrm{PGE}_{2}$ and matrix metalloproteinase 9, which also may be involved in disruption of the BBB. Permeability of the BBB could be increased as well by pro-inflammatory cytokines induced by $S$. suis and produced by BMECs and/or by suilysin produced by the pathogen. Finally, S. suis breaches the BBB function and integrity by killing its cells, mostly by necrosis and less often by apoptosis $[1-3,7,11]$.

Liu et al. [12], using primary porcine brain microvascular endothelial cells and astrocytes cell culture model, identified a total of 14 potential virulence factors contributing to $S$. suis type 2 adherence to and invasion of the BBB. Twelve of these factors, mostly enzymes (such as DNA-directed RNA polymerase subunit beta, $\mathrm{O}$-acetylhomoserine sulfhydrylase, a collagenase-like protease, IS630-Spn1 transposase Orf2, COG0642 signal transduction histidine kinase), were reported for the first time to potentially contribute to $S$. suis breaking into the BBB. Recently, Kong et al. [13] demonstrated that the interaction of factor H-binding protein of S. suis with the host's globotriaosylceramide cell receptor promotes the traversal of the bacterium across the human $\mathrm{BBB}$ and the development of meningitis $[8,13]$.

In pigs, septicaemia with sudden death, meningitis, arthritis, endocarditis, and, as a secondary agent, pneumonia, are the most common features of $S$. suis disease. In humans, meningitis and septic shock (including streptococcal toxic shock-like syndrome) are the most common expressions of the disease, although many other clinical manifestations have been described [6].

Inflammation. Clinical presentations of S. suis infection vary from asymptomatic bacteraemia to fulminant systemic disease with septic shock similar to Gram-negative sepsis, which suggests a massive inflammation process. Clinical signs of meningitis in pigs and humans have also been associated with inflammation in the CNS. Thus, it is evident that inflammation plays a key role in the pathogenesis of both systemic and CNS infections caused by S. suis [1]. The pathogen induces the overproduction of pro-inflammatory cytokines and the recruitment and activation of different leukocyte populations, causing acute inflammation of the CNS [11].

Inflammatory reaction induced by S. suis at the CNS may play an important role in the pathology of meningitis. S. suis induces the release of arachidonic acid by BMECs which increases the ability of bacteria to penetrate the CNS and to modulate local inflammation $[2,11]$. Gottschalk et al. [11] demonstrated that $S$. suis induces the release of proinflammatory cytokines by human BMECs and up-regulates the expression of adhesion molecules on human monocytes, with consequent increased adherence of S. suis-activated monocytes to endothelial cells.

Streptococcus suis can also evoke a fulminant inflammatory reaction of the brain which takes place through activation of microglial cells, astrocytes, and possibly other cell types by the cell wall and secreted (such as suilysin) components of the bacterium. Inflammation of the CNS leads to intracranial complications, including brain oedema, increased intracranial pressure and cerebrovascular insults [2].

According to Strangmann et al. [14], involvement of the $8^{\text {th }}$ cranial nerve, a characteristic of $S$. suis meningitis that occurs in the days following the onset of the meningeal syndrome, can lead to permanent deafness. Another mechanism is proposed by Gottschalk et al. [11] who expressed an opinion that the accumulation of inflammatory cells observed around the vestibular-cochlear $\left(8^{\text {th }}\right.$ cranial $)$ nerve in S. suis meningitis is insufficient to impede normal neural conduction. According to these authors, rather cochlear sepsis, resulting from passage of the organism from the sub-arachnoid space to the perilymph via the cochlear aqueduct, might be primarily responsible for the hearing loss complicating bacterial meningitis caused by $S$. suis. This view has been supported by the results obtained by Dominguez-Punaro 
et al. [15], who observed that mice infected with a high doses of $S$. suis bacteria developed sustained bacteraemia, with an increase in the permeability of the blood-labyrinth and blood-brain barriers, resulting in suppurative labyrinthitis and meningitis.

\section{INTERACTION WITH HOST CELLS AND TISSUES}

Interaction with leukocytes and macrophages in the blood. A major first line of host defence against S. suis is phagocytic killing by neutrophils. Accordingly, infiltrations with large number of neutrophils are typically found in respective lesions, such as in meningitis. In the absence of opsonising antibodies, virulent $S$. suis serotype 2 strains are not efficiently killed by porcine neutrophils, suggesting that the pathogen has evolved strategies to evade the intra- and extracellular antimicrobial activity of neutrophils [3].

As mentioned earlier, S. suis evades phagocytosis by host neutrophils, monocytes and macrophages thanks to the protection provided by CPS. The S. suis types 2 and 14 CPS were found to prevent phagocytosis by destabilizing lipid microdomains (rafts) of phagocytes which hinders accumulation of lactosylceramide (a bioactive member of the glycosphingolipid family) at the interaction points between $S$. suis and the cell membrane, with consequent inhibition of key signaling pathways involved in phagocytosis and cell activation, including nitric oxide (NO) production [7, 16-18].

The resistance of $S$. suis to phagocytosis is multifactorial, not only confined to CPS. $N$-deacetylation of the peptidoglycan reduces killing by neutrophils, probably by providing the bacterium with enhanced resistance against the action of lysozyme. D-alanylation of the LTA (lipoteichoic acid) contributes to enhanced resistance to host antimicrobial peptides and to resistance to neutrophil killing. S. suis produces a cell-wall-anchored DNase, specific for singleand double-stranded linear DNA, suggested to participate in NETs (neutrophil-extracellular trap) breakdown. The bacterium also secretes the serine protease SspA, capable of degrading IL-8, a major chemo-attractant of neutrophils. Secreted suilysin has been shown to be toxic to phagocytes and also to interfere with complement activity. If internalized, S. suis may use SOD (superoxide dismutase) and the arginine deiminase system to resist the intracellular environment [2].

Interactions between $S$. suis and human leukocytes are similar to those reported with swine or mouse phagocytes. Although the route of bacterial entry might differ between human and pigs, the pathogenesis of infection might occur through similar mechanisms [1].

Ye et al. [19] proposed the following 2-stage model of the pathogenesis of severe human disease caused by the epidemic S. suis strain ST7: In stage 1, on entry of the pathogen into the bloodstream, bacterial cell-wall components interact with the host immune system via pattern-recognition receptors, such as TLR-2 (Toll-like receptor 2) and CD14 (cluster of differentiation 14), and probably by the 2-component signal-transduction system. In stage 2 , these host-pathogen interactions trigger a burst of pro-inflammatory cytokines, which may contribute to the development of toxic shock.

De Buhr et al. $[20,21]$ demonstrated that S. suis induced the formation of the neutrophils extracellular traps (NETs) in the porcine blood in vitro. Bacteria were entrapped but not killed by these NETs [20]. To evade the traps, S. suis produces at least 2 extracellular nucleases degrading the DNA of the NETs [21]. More recently, these authors found that NETs are also formed in vivo in the cerebrospinal fluid (CSF) of piglets with meningitis caused by $S$. suis, and are protected by antimicrobial peptides against bacterial nucleases [22].

Interaction with dendritic cells. Dendritic cells (DCs) are powerful antigen presenting cells that occur mostly in the tissues that are in contact with the external environment, and initiate the immune response against pathogens. After the capture and processing of antigens, DCs undergo a maturation process characterized by the production of cytokines and upregulation of co-stimulatory molecules, and then migrate to the adjacent lymphoid organs where they activate T cells. Thus, DCs represent a crucial link between innate and adaptive immunity and interactions between DCs and pathogens can strongly influence the outcome of a disease $[23,24]$.

Lecours et al. [23, 25], in their pioneer studies on the interactions between Streptococcus suis and bone marrowderived swine DCs, examined the role of DCs in porcine streptococcosis. Their studies provided evidence that a multimodal recognition involving a combination of different receptors is essential for DC effective response to $S$. suis. The crucial mechanism for DC activation and maturation in response to $S$. suis infection is the myeloid differentiation factor 88 (MyD88) signaling. This mechanism is initiated by interactions between the DCs receptors: TLRs (Toll-like receptors) and NODs (Nucleotide-binding oligomerization domain), and the multiple $S$. suis cell wall receptors. The association of activated DCs receptors with the MyD88 adaptor molecule initiates an intracellular signaling cascade that induces the secretion of pro-inflammatory cytokines IL- $1 \beta$, IL- 6 and TNF- $\alpha$, the T cell-activating cytokines IL-12p70 and IL-23, the regulatory cytokine IL-10, and the chemokines CXCL1 (believed to be important in the trafficking and activation of neutrophils) and CXCL10. The MyD88-dependent signaling also induces the expression of co-stimulatory cell-surface molecules through the activation of transcription factors, including NF- $\kappa \mathrm{B}$.

Signaling is mainly initiated through Toll-like 2 receptors (TLR2). Toll-like 9 receptors (TLR9), in conjunction with TLR2 and NOD2, are also involved in cell activation. Lecours et al. [25] expressed an opinion that also other receptors, including other TLRs (such as TLR3), may mediate activation and maturation of DCs by $S$. suis and participate in the activation of the immune response. TLRs do not seem to be involved in S. suis phagocytosis by DCs, which is independent from signaling through MyD88.

In a further study on the DCs-mediated response to $S$. suis with the use of mouse model, Lecours et al. [26] demonstrated that the $S$. suis-stimulated DCs activated $\mathrm{CD} 4^{+} \mathrm{T}$ cells which developed Th1 (T helper type 1) response, manifested by the increased production of TNF- $\alpha$, IL-6, IFN- $\gamma$, CCL3, CXCL9, and IL-10 cytokines.

Recently, Lemire et al. [27] demonstrated in a mouse model that DCs stimulated with $S$. suis activate NK cells, e.g. large granular lymphocytes described as 'natural killer cells' that play a crucial role in the innate immune response against microorganisms. Activated NK cells produce IFN- $\gamma$ which, in turn, initiates a DC-NK crosstalk mainly dependent on cell-to-cell contact. This interaction results in enhanced cytokine production that results in the amplification of 
deleterious inflammatory response, rather than the control of bacteraemia levels.

In another recent study, Martelet et al [28] demonstrated that the activation of DCs with various $S$. suis antigens (enolase, CPS, and its conjugated form) was increased by the concurrent use of commercial adjuvants, and polarized towards type 1 or type 2 immunologic response. The authors concluded that the applied DC culture model can be used to test the future $S$. suis vaccine candidates, and accelerate the design of promising subunit vaccines against this pathogen.

The CPS was shown to modulate most interactions with DCs by protecting bacteria against phagocytosis, reducing the level of cytokine production and preventing the surface expression of co-stimulatory molecules. Lecours et al. [23] observed that the unencapsulated $S$. suis mutant adheres to and is internalized by DCs at markedly higher numbers than the wild-type encapsulated strain (Fig. 1). However, the presence of CPS does not protect the bacteria against intracellular killing [23]. Lecours et al. [26] demonstrated that the $S$. suis CPS interferes with $\mathrm{CD} 4^{+} \mathrm{T}$ cell activation, while having no impact on $\mathrm{T}$ cell proliferation. According to Lemire at al. [27], the S. suis CPS impairs NK cell activation.

Interaction with microglia and other brain cells. Microglia are resident macrophage cells of the CNS that act as the first and main line of active immune defence and play an important role in keeping CNS homeostasis [29]. Other brain cells include the neural network and supporting macroglia, among which the most numerous are astrocytes, also implicated in the immune response of CNS [30].

The interaction of $S$. suis with murine CNS cells has been thoroughly studied by Dominguez-Punaro et al. [31, 32]. In the first study in vivo [31], they demonstrated for the first time that microglia and, to a lesser extent, astrocytes, were activated by the $S$. suis invasion of CNS and that these cell types were probably the sources of cytokine induction in the brain parenchyma. A general analysis of cytokine patterns suggests that infection with $S$. suis serotype 2 induces a Th1type immune response.

S. suis was able to induce strong transcriptional activation of genes encoding pro-inflammatory molecules in various structures of the brain, mainly in myeloid cells located in affected cerebral structures. The detected molecules included TLR2, TLR3, CD14, NF- $\kappa$ B, IL-1 $\beta$, CCL2/MCP-1, and TNF- $\alpha$. The authors demonstrated in vitro that TLR2 plays an important role in the recognition of S. suis through a MyD88-dependent mechanism, similarly as evidenced by Lecours et al. [25] in the case of activated dendritic cells. Early transcriptional activation of TLR2, CD14, and inflammatory cytokines in the choroid plexus and cells lining the brain endothelium suggests that these structures are potential entry sites for the bacteria into the CNS. Induction of the cytokine cascade markedly increases the severity of meningitis, and in some cases encephalitis, caused by $S$. suis [31].

In the second study performed in vitro, DominguezPunaro et al. [32] confirmed the former results obtained in vivo and demonstrated that the murine microglial cells are critically implicated in the CNS inflammatory response induced by S. suis. They evidenced that S. suis induces production of pro-inflammatory cytokines and chemokines (TNF- $\alpha$, IL-1 $\beta$, IL-6, MCP-1, and IP-10) by murine microglia, and TLR2 gene expression, but not the expression of TLR1, TLR4, TLR6, and TLR9 genes. S. suis infection of murine microglia also induces the activation NF- $\kappa \mathrm{B}$, which is one of the most prominent transcription factors involved in the inflammatory response, and the expression of inducible nitric oxide synthase (iNOS) and NO release which might lead to tissue destruction and degeneration. Moreover, S. suis infection activates in murine microglia different MAPK (mitogen-activated protein kinase) signaling pathways, leading to the production of different pro-inflammatory mediators that play essential roles in the host response to pathogens and deterioration of brain tissues. Thus, activated microglia are implicated in neurodegeneration resulting from bacterial meningitis.

The above-cited results were further confirmed by Zheng et al. [33] who infected the BV2 cell line, a substitute for primary microglia cells, with the $S$. suis highly pathogenic strain ST7 (sequence type 7) which caused a major epidemics in China in 2005, and with the pathogenic strain ST1. They proved that the ST7 strain induced a greater microglial inflammatory response compared to ST1, as shown by a higher pro-inflammatory cytokine production, as well as by higher activation of MAPK signaling pathways and several transcription factors. Finally, the authors showed that signal transducer and activator of transcription 3 (STAT-3) was involved in the development of meningitis induced by $S$. suis ST7 infection.

More recently, Seele et al. [34] established the primary mouse astrocyte-microglial cell co-cultures and proved that astrocytes and astrocyte supernatants increased the interaction of microglial cells with S. suis and NO release.

Stimulation of the excessive inflammatory response. The course of infection process caused by Streptococcus suis in swine, mice and humans is a good example for confirmation of the thesis that the host's immune system may play an important role, not only in the development of protection against disease, but also in the pathology caused by the invading pathogen [11]. The interaction of S. suis with innate and adaptive immune mechanisms of the host triggers a burst of pro-inflammatory cytokines, which may contribute to the development of toxic shock in humans referred to as the Streptococcal Toxic Shock-Like Syndrome (STSLS) $[4,19]$.

The overproduction of pro-inflammatory cytokines is initiated by the binding of the $S$. suis molecules to TLR2 or CD14 receptors of immunocompetent cells, such as leukocytes in the blood and lymph, microglia in NCS, and macrophages and dendritic cells in other organs and tissues $[23,32]$. The excessive amounts of cytokines induce an acute inflammatory reaction manifested by massive infiltrations of leukocytes, mainly neutrophils, into lesions $[3,11]$.

The cytokine cascade induced by $S$. suis in the early phase of infection includes: tumour necrosis factor $\alpha$ (TNF- $\alpha$ ), interleukins IL-1 $\beta$, IL-6, IL- 8 and IL-12, interferon- $\gamma$ (IFN- $\gamma$ ), and the chemoattractants (chemokines) CCL2/MCP1, CXCL1/KC, and CCL5/RANTES. Most cytokines are induced by S. suis cell wall components, whereas CCL2/MCP1 is induced mainly by the CPS $[2,11,19,31]$. Dominguez et al. [31] expressed the opinion that abundant release of these cytokines in mice 24 hrs post-infection with S. suis might be responsible for the sudden death of $20 \%$ of the animals.

Ye et al. [19], during the epidemics in China in 2005, observed that the serum levels of cytokines (IL-1 $\beta$, IL-6, IL-8, IL-12, IFN- $\gamma$, TNF- $\alpha$ ) during the early phase of the disease, were significantly more elevated in patients with STSLS, 

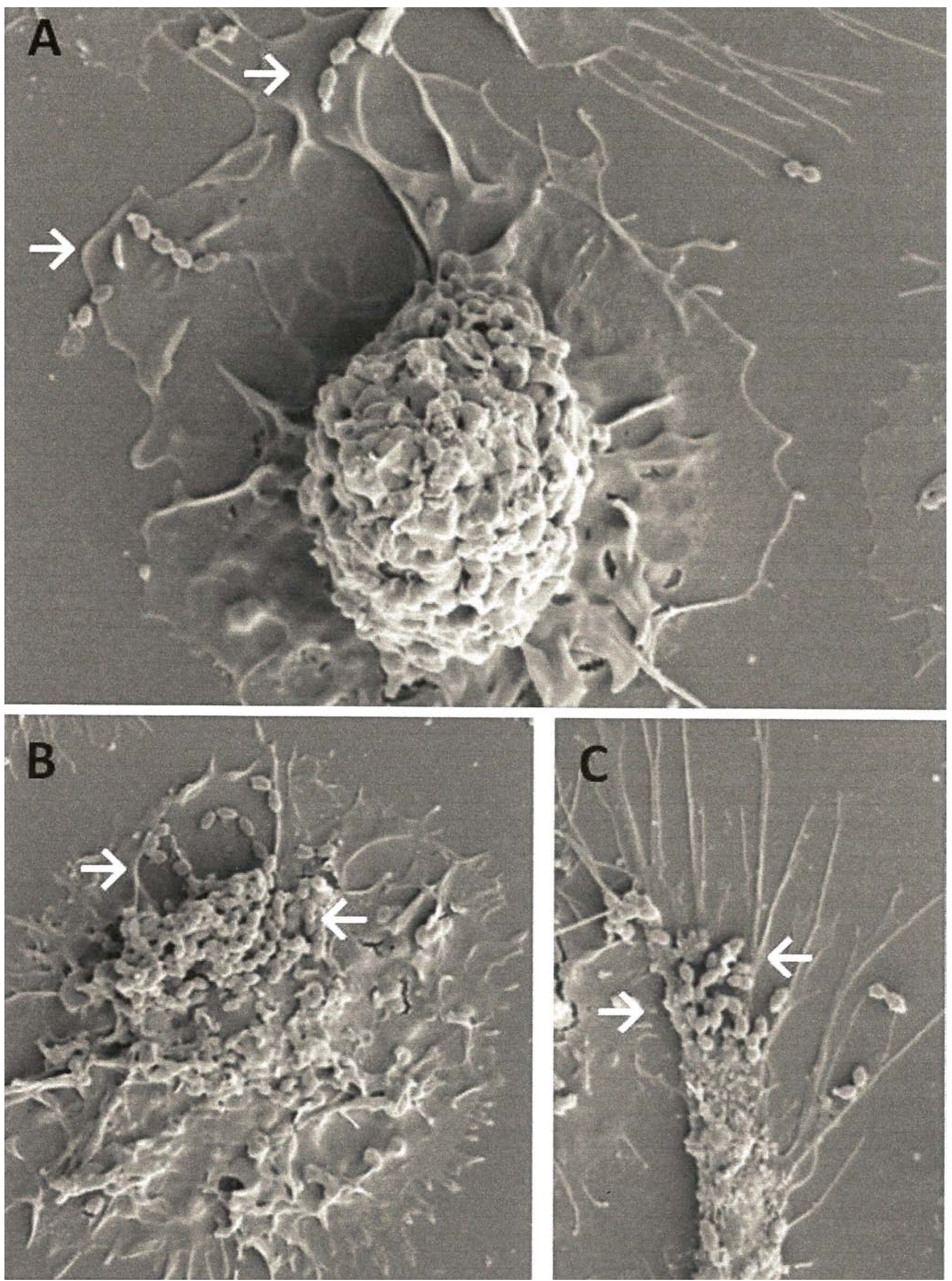

Figure 1. Scanning electron micrographs showing interactions between dendritic cells (DCs) and S. suis. DCs were incubated with the S. suis encapsulated wild-type strain (WT) or the non-encapsulated S. suis mutant (CPS-) for 2 hrs. (A) DCs incubated with S. suis WT strain show very few cocci on the cell surface. (B-C) DCs incubated with the S. suis CPS- mutant show much more cocci adhering to the cells. White arrows show bacterial cells. Original magnification $5,000 \times$. According to Lecours et al. [23]. 
compared to those with meningitis. In a mouse model, these authors demonstrated that the levels of pro-inflammatory cytokines were the highest in animals infected with the most pathogenic strain ST7 that caused the epidemics, lower in those infected with the pathogenic strain ST1, and lowest in those infected with the least pathogenic strain - ST25. In conclusion, the authors expressed an opinion that stimulation of the production of massive amounts of pro-inflammatory cytokines by highly pathogenic strains ST7 and ST1 is the main reason for shock syndrome observed in severe humane cases of porcine streptococcosis [19].

Zhao et al [35] and Jiang et al [36] recently indicated a type IV-like secretion system (T4SS-like system) harboured within the S. suis $89 \mathrm{~K}$ Pathogenicity Island (PAI), and its main component VirD4 factor, as mechanisms responsible for the excessive cytokine burst and STSLS pathogenesis.

\section{VIRULENCE FACTORS}

Introduction. In the early studies on pathogenesis and virulence of Streptococcus suis performed in 1990s, the CPS (capsular polysaccharide) [37,38], 2 proteins defined as MRP (muramidase-released protein) and EF (extracellular factor) [39], and secreted thiol-activated, membrane-damaging toxin suilysin (haemolysin) [40, 41], have been identified as virulence factors. Since then, a multitude of the novel virulence factors have been identified, approximating 23 in 2007 [11], 71 in 2012 [2] and 100 in 2017 [10]. Of the first identified factors, the most important is CPS which confers antiphagocytic properties to the bacteria $[1,23,25,37,38]$, and has been defined by Gottschalk et al. [1] as 'the single most critical virulence factor'. Suilysin is other important virulence factor that is toxic for various cell types of the host, and protects against complement-mediated phagocytosis [1], while the role of MRP and EF in the pathogenesis of the disease caused by S. suis is controversial, and according to Fittipaldi et al. [2] they should be defined rather as 'virulence markers' than 'virulence factors'. In their thorough review of the $S$. suis virulence factors, the cited authors expressed an opinion that a universal virulence factor for all S. suis strains probably does not exist [2].

In a recent critical review, Segura et al. [10] reported that from over $100 \mathrm{~S}$. suis 'putative virulence factors or traits', at least 37 were claimed to be 'critical' for virulence. The authors regard this number as an overestimate, mainly due to the large genetic diversity of $S$. suis and to the fact that pathogenesis of the disease caused by this organism is a complex, multifactorial process. As a result, many of the virulence factors which are redundant (e.g. perform similar function) were described under different names. To avoid confusion, the authors proposed standardization of the animal models and research methods aimed at assessing the virulence of S. suis [10]. As most of the hitherto performed research in this area has been conducted with the most pathogenic S. suis type 2 strains, there is a need for a more detailed study on the virulence of the remaining types of this pathogen.

Feng et al. [4] classified temporarily the S. suis virulenceassociated factors into the following 4 groups:

1) surface/secreted elements;

2) enzymes, including proteases;

3) transcription factors/regulatory systems;

4) others (transporters/secretion systems).
The most important factors of each group are reviewed below.

Capsular polysaccharide as a major virulence factor of Streptococcus suis. As already mentioned, the capsular polysaccharide (CPS) of S. suis is considered a major virulence factor as it efficiently protects bacteria against phagocytosis and cloaks cell wall antigens, enabling evasion of the immunity response of the host $[1,10,16,18,42]$. On the other hand, it hampers adhesion to and invasion of the host's cells, release of inflammatory cytokines and biofilm formation $[7,16,42]$. Also, the CPS cannot be regarded as a virulence marker, as most of avirulent strains are encapsulated [1].

The $S$. suis CPS shows a marked diversity, and its antigenicity is a basis for division of the species into 35 (sero)types. CPS production is regulated by the capsular polysaccharide biosynthesis (cps) gene clusters which differ depending on the type. Okura et al. [43] demonstrated that 35 type-specific gene clusters occupy different chromosomal loci and vary in length between 15,274-40,198 bp (base pairs).

The CPS of S. suis type 2 is composed of the monosaccharides glucose, galactose, $\mathrm{N}$-acetyl-glucosamine, and rhamnose arranged into a unique repeating unit containing a sidechain terminated by sialic acid ( $N$-acetylneuraminic acid, coded by the genes neuA to neuD) which is $\alpha$-2,6-linked to galactose. Sequencing of the $S$. suis most pathogenic type $2 c p s$ locus revealed 17 potential genes involved in sugar transfer, polymerization of CPS, and synthesis of sialic acid [16]. Among the genes in the cluster, cps $2 J$ serves as a molecular marker for serotypes 2 and $1 / 2$ [44].

The ambiguous role of the S. suis CPS was noted by various authors. Domínguez-Punaro et al. [32] demonstrated in the murine microglial cells model that while encapsulated S. suis strain protected bacteria against the immune response and induced enhanced expression of Toll-like receptor 2 and protein kinase, the CPS-deficient mutant increased the phosphorylation profiles, production of nitric oxide and activation of the proinflammatory transcription factor NF$\kappa B$. Segura [16] expressed an opinion that the S. suis CPS dramatically impairs the production of most cytokines and surface expression of co-stimulatory molecules by infected dendritic cells (DCs). As a result, phagocytosis is inhibited. This view has been confirmed by Calzas et al. [45] who studied the effects of the purified CPS preparations of S. suis types 2 and 14 on bone marrow-derived murine DCs, and found a generally low immunologic response, except for a significantly increased production of the pro-inflammatory chemokines CCL 2 and CCL 3 via Toll-like receptor 2 (TLR2), and myeloid differentiation factor 88 (MyD88)-dependent pathways. In more recent studies conducted on mice and pigs in vivo, Calzas et al. $[46,47]$ noted a weak IgM antibody response and no IgG antibody response to CPS preparations obtained from S. suis strains belonging to types 2 and 14 . Summarizing, the $S$. suis CPSs are generally considered poorly immunogenic.

It is evident from the above-presented results that the presence or absence of CPS could be both advantageous and disadvantageous for S. suis strains. The CPS-possessing strains are resistant to immune response of the host, but cannot efficiently penetrate the host's cells, whereas CPSdeficient strains which are prone to phagocytosis can successfully adhere to the host's cells and propagate therein. Thus, both phenotypes are complementary in action and can assist each other in the successful progress of the infection 
process. Based on these facts, Segura et al. [10] recently formulated a hypothesis that $S$. suis evolved transcriptional mechanisms that enable reversible mutations in cps genes, resulting in an off-on switching of CPS expression. Such mechanisms would enable an easy transition between the asymptomatic and symptomatic form of the disease and a successful colonizing of various tissues and organs of the host by the bacterium.

The sound hypothesis of Segura et al. [10] conforms to the results obtained by other authors in Japan, mostly those experimenting on S. suis type 2 strains isolated from pigs with endocarditis. Auger et al. [48] demonstrated that the unencapsulated porcine $S$. suis strain retrieved the capsule after in vivo passages in mice. Lakkitjaroen et al. [44] showed that $34 \%$ of cps $2 J$-positive S. suis isolates from porcine endocarditis lost their capsules, and expressed an opinion that the loss of the capsule, because of increased ability to adhere to porcine and human platelets, appears to be beneficial to the pathogen in the course of disease. The authors found that a single-nucleotide substitution and frameshift mutation in 2 glycosyltransferase genes ( $c p s 2 E$ and $c p s 2 F$ ) were the main causes of the capsule loss, having been observed in at least $74.4 \%$ of examined isolates. Meaningful results were obtained by Tohya et al. [49] who found the coexistence of both encapsulated and unencapsulated S. suis isolates in 26 out of 59 (44\%) endocarditis (heart valve) samples taken from pigs. The data showed that each pair of isolates had one or more unique non-synonymous mutation in the cps gene, and the encapsulated and unencapsulated isolates from the same samples were closest to each other. Pair-wise comparisons of the sequences of $c p s$ genes identified insertion/deletions (indels) ranging from $1-10^{4} \mathrm{bp}$ in different cps genes of unencapsulated isolates. The authors concluded that the results demonstrate the coexistence of bacterial clones of dual phenotypes (encapsulated and unencapsulated) in one animal host that arose independently in each farm by means of spontaneous mutations in cps genes. Such coexistence increases the chance for successful infection progress by the complementary 'cooperation' of both clones.

The sialic acid component of the $S$. suis CPS is related to many biological activities of the capsule, the more so as the presence of capsular sialic acid is a unique characteristic of Streptococcus suis and Streptococcus agalactiae among Gram-positive bacteria [16, 47]. Roy et al. [18] demonstrated that lack of the sialic acid synthesis gene prevented CPS production in the $\Delta n e u 14 C$ mutant of a S. suis type 14 strain (Fig. 2). Sialic acid is most likely responsible, at least in part, for the antiphagocytic properties of the CPS $[1,16]$. This component has also been implicated in the modulation of the interactions with different host cells, including adherence, without phagocytosis, of $S$. suis to monocytes, suggesting a 'modified Trojan horse' hypothesis, in which the pathogen would travel in the bloodstream externally associated with these phagocytic cells. On the other hand, the presence of sialic acid in the S. suis CPS creates a negative charge on the bacterial surface. Therefore, it is likely that a host cell surface negative charge and the sialic acid containing CPS form a biophysical repulsion strong enough to reduce adhesion of encapsulated S. suis to some host cells [7].

The conserved 2-6 linked sialic acid terminal capsular moiety found in S. suis serotypes 2 and 14 is similar to sugar epitopes widely displayed on the surface of all mammalian cells. This molecular mimicry enables $S$. suis to evade the recognition by the immune system of the host [2].

The capsular sialic acid also plays a major role in the interaction of S. suis with other pathogens. Wang et al. [50] demonstrated that the sialic acid moiety present in the CPS of $S$. suis serotype 2 directly interacts with swine influenza virus $\mathrm{H} 1 \mathrm{~N} 1$, leading to increased bacterial adhesion to, invasion of, and activation of swine tracheal epithelial cells. Cells pre-infected with $\mathrm{H} 1 \mathrm{~N} 1$ virus showed bacterial adhesion and invasion levels that were increased more than 100-fold, compared to those of normal cells. This leads to exacerbation of influenza infection.

Other virulence factors. In the temporary classification by Feng et al. [4], the first group of S. suis virulence factors consists of the surface and secreted elements. For this group, at least 17 genes/gene clusters have been determined thus far to contribute to bacterial pathogenicity. They include the following factors (acronyms in square brackets, gene names in round parentheses and italic):

- capsular polysaccharides [CPS] (cps);

- extracellular protein factor [EPF] (epf);

- fibronectin binding adhesin [Fbps] ( $f b p s)$;

- muramidase released protein [MRP] $(m r p)$;

- a protein of $38 \mathrm{kDa}$ localized on bacterial surface $(38 \mathrm{kDa})$;

- secreted thio-activated heamolysin (suilysin) [SLY] (sly) which is not only a toxic factor for various cell types, but also interferes with complement-mediated phagocytosis and killing;

- surface-associated subtilisin-like serine protease [SspA] $(\operatorname{SspA})$ that modulates cytokine secretion by macrophages contributing to the process of meningitis;

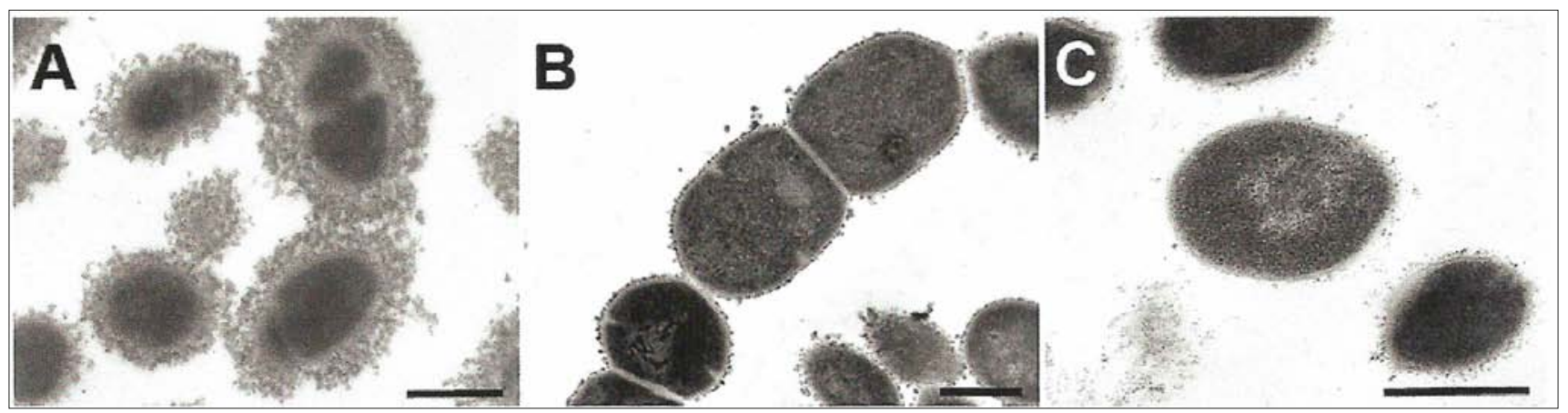

Figure 2. Transmission electron micrographs showing the expression of capsular polysaccharide (CPS) by the wild-type strain of Streptococcus suis serotype 14 and its derived mutants: capsule-deficient mutant $(\triangle c p s 14 B)$ and a mutant devoid a gene coding for uridine diphospho-N-acetylglucosamine 2-epimerase which is involved in sialic acid synthesis ( $\Delta$ neu14C). The CPS was labelled with polycationic ferritin. The wild-type strain, DAN13730 (A), is surrounded by a thick capsule, whereas the $\triangle c p s 14 B$ and $\triangle$ neu14C mutant strains (B and C, respectively) are non-encapsulated. Bars $=0.5 \mathrm{~mm}$. According to Roy et al. [18], with permission. 
- a histidine triade immunogenic cell surface protein $[\mathrm{HtpS}]$ (htpS);

- Sat surface protein (sat);

- a novel serum opacity factor [OFS] (ofs);

- surface antigen protein [SAO] (sao);

- sortase A (SrtA), catalyzing cell wall sorting reaction;

- pili, hair-like appendages composed of pilin proteins that are active in biofilm formation.

Some factors from this group (such as $S s p A$, ofs, sao) are LPXTG-motif containing proteins which are covalently anchored to the cell wall peptidoglycan with sortase (SrtA). Pilins are covalently linked via motifs closely resembling the LPXTG sequences. Recombinant Sat protein, and HtpS, a member of histidine triad protein family, can confer effective immuno-protection against $S$. suis type 2 infections to mice, implying that they are a vaccine molecule candidates $[1,2,4,7]$.

The second group is composed of enzymes, including proteinases. Feng et al. [4] suggested that no less than 22 bacterial enzymes might be implicated in the manifestation of S. suis virulence. Among significant enzymatic virulence factors are following:

- GlnA, glutamine synthetase $(g \ln A)$;

- Gdh, glutamate dehydrogenase $(g d h)$;

- enolase (eno) catalyzing dehydration of 2-phosphoglycerate to phospho-enolpyruvate which also acts as adhesin binding to host fibronectin;

- enzyme catalyzing lipoteichoic acid (LTA)-d-alanylation (dltA);

- peptidoglycan N-acetylglucosamine deacetylase ( $p g d A)$;

- inosine 5-monophosphate dehydrogebase [Impdh] (impdh);

- N-acetylneuraminic acid (sialic acid) synthetase (neuB);

- UDP N-Acetylglucosamine 2-Epimerase (neuC) that was found to be essential for capsule production;

- glyceraldehyde-3-phosphate dehydrogenase $(G A P D H)$ that catalyzes glycolysis and acts as adhesin;

- DNase (112 kDa) degrades DNA, enables evasion of the host's immune response;

- IgA1 (IgA1) protease cleaving immunoglobulin A;

- superoxide dismutase (sod) that catalyzes partitioning of the superoxide radical;

- adenosine synthase (Ssads) which functions as an effector in evasion of granulocyte-mediated innate immunity;

- LuxS or S-ribosyl homocysteinase (luxS) catalyzing synthesis of auto-inducer 2 (AI-2) utilized in interspecies quorum sensing (QS) communication system regulating virulence, capsule biosynthesis and biofilm formation in S. suis (QS is a feedback system regulating life functions of bacteria and interrelations between bacterium and host depending on density of bacterial colonizer, which is steered by a diffusible signal molecule, in this case AI-2, targeting various functions).

Some of these enzymes are directly or indirectly related to synthesis and/or modification of bacterial surface structure which is critical for successful invasion and entry of pathogens into host cells. These enzymes include an enzyme catalyzing lipoteichoic acid (LTA)-d-alanylation (dltA), and peptidoglycan $\mathrm{N}$-acetylglucosamine deacetylase ( $p g d A$ ) that significantly contribute to $S$. suis pathogenicity $[2,4,51$, 52]. Seele et al. [53, 54] described a novel Ide(Ssuis) protease degrading porcine immunoglobulin $\mathrm{M}$ that functions as a complement evasion factor, enabling bacterial survival in porcine blood during the early adaptive (IgM-dominated) immune response. The authors found that this protease could be used as a potential vaccine component as it protected piglets against disease caused by the invasive type $2 S$. suis strain [55]. In another study by this team, Spoerry et al. [56] described a novel IgG-degrading protease, also with some prospects for use as a potential vaccine component.

The third group are transcriptional factors/regulators that comprise at least 17 pleiotropic regulators which were identified as being involved in the modulation of $S$. suis virulence. They include transcription factors, two-component signal transduction (TCS) systems and orphan regulators. Transcription factors include:

- AdcR ( $a d c R)$, a regulator controlling zinc transport in S. suis;

- catabolite control protein A ( $c c p A)$, a sugar metabolism regulator;

- ArgR $(\operatorname{argR})$, an ADS-associated repressor of the ArgR/ AhrC arginine family, which regulates expression of $\operatorname{arc} A B C$ operon encoding an arginine deiminase system catalyzing the conversion of arginine to ornithine, ammonia and carbon dioxide, which increases the survival of $S$. suis under acidic conditions;

- Rgg $(r g g)$ transcription factor that plays multiple roles in bacterial metabolism;

- Fur (fur), an iron uptake regulator;

- PerR (perR), an $\mathrm{H}_{2} \mathrm{O}_{2}$-responsive Fur-like regulator, which determines bacterial virulence through regulating expression of both $d p r$, a Dps-like peroxide resistance protein-encoding gene, and metQIN encoding a methionine transporter.

Among the 15 putative TCS systems identified in the Chinese epidemic, S. suis strain, 5 (salK-salR, ciaR-ciaH, ihkihr, virR-virS, nisK-nisR) are correlated with manifestation of strong virulence. Of which, the most important is the salK-salR system present in the $89 \mathrm{~K}$ pathogenicity island, where it regulates bioactive lantibiotic suicine production. The deletion of this TCS system resulted in significant downregulation of 26 genes expression level, and increased S. suis susceptibility to polymorphonuclear leukocyte (PMN)mediated killing. Orphan regulators comprises CovR, RevSC and RevS factors $[2,4,7]$.

The fourth group of transporters/secretion systems comprises following factors:

- VirA (virA) present in virulent S. suis type 2 strains;

- Trigger factor (Tig) that is a virulence determinant which acts by controlling expression of a collection of known virulence factors, such as cps, $m r p$, and $s l y$;

- FeoBA ( feoBA) encoding an iron transporter system;

- Type IV like-secretion system (T4SS-like system) present in the $89 \mathrm{~K}$ pathogenicity island that consists of 2 elements: VirD4-89K/VirB4-89K (virD4-virB4), implicated in the pathogenesis of Streptococcal Toxic Shock-Like Syndrome (STSLS) $[4,35]$.

This system, proposed as a new T4SS subgroup (Type-IVC secretion system), contains a channel enabling secretion of proteins and DNA molecules across the cell envelope [36].

The knowledge of $S$. suis virulence factors and their role in the diseases caused by this bacterium has been steadily extended by recent publications, mostly performed in China. 
Jiang et al. [36] demonstrated that the VirD4 factor, a key component of the S. suis T4SS-like system, contributes to STSLS pathogenesis, possibly via its anti-phagocytic activity, upregulation of its expression upon oxidative stress and its involvement in increased secretion of PrsA (peptidylprolylisomerase, a novel protein) as a cell death inducer and pro-inflammatory effector. Gao et al. [57] proved that a Glucose-inhibited division protein (GidA) coded by gene gidA, is a tRNA modification enzyme that plays an important role as a translational regulator for the expression of particular proteins involved in the growth, cell division, capsule synthesis, and virulence in Streptococcus suis type 2. Feng et al. [58] clearly demonstrated that CodY, a global regulatory protein in most low $\mathrm{G}+\mathrm{C}$ Gram-positive bacteria, modulates cellular growth and virulence in $S$. suis type 2 , increases its resistance to phagocytosis and survival in blood, and modulates global gene expression, especially genes involved in metabolism and capsule synthesis - in particular those engaged in the sialic acid synthesis in the capsule. S. suis mutant devoid of $\operatorname{cod} Y$ gene had a significantly thinner capsule compared to the parent strain (Fig. 3). Ji et al. [59] identified a novel virulence-associated protein E coded by $v a p E$ gene as an important factor associated with the pathogenicity of S. suis type 2. Zhu et al. [60] demonstrated that (p)ppGpp synthetases that mediate adaptive response of bacteria), could regulate the growth and pathogenicity of S. suis. Tan et al. [61] demonstrated that MsmK, an ATPase of ATP-binding cassette (ABC) transporters, is a multifunctional factor that not only plays an important role in carbohydrate utilization by $S$. suis, but also contributes to its resistance to blood killing, phagocytosis, and osmotic stress, increases haemolytic activity and biofilm formation and promotes ability of the pathogen to infect the hosts.

Yuan et al. [62] described a new 1910HK/RR two-component system that is essential for the virulence of Streptococcus suis type 2 , and for the survival of the pathogen in human blood. Zhang et al. [63] demonstrated that the eukaryote-like serine/threonine kinase (STK), encoded by a single-copy stk gene, is crucial in stress response and virulence and plays an important role as a central regulator of the growth, cell division and metabolism of S. suis type 2. A mutant strain devoid of $s t k$ gene revealed a slower cell division rate, resulting in much longer cell chains compared to the parent strain (Fig. 4). Ferrando et al. [64] showed that Streptococcal Adhesin P (SadP) contributes to Streptococcus suis adhesion to the human and porcine intestinal epithelial cells (IEC) and to its translocation across the human IECs. According to authors, SadP is a bifunctional protein with host-cell adhesion and host immune evasion properties. Auger and Gottschalk [8] regard it as identical with H-binding factor. Lai et al. [65] identified in the S. suis type 9 strain the presence of a type VII secretion system (T7SS) putative substrate EsxA that contributes to bacterial virulence. Xu et al. [66] identified in the $S$. suis type 2 strain the manganese efflux system MntE which controls the manganese homeostasis that is important for the pathogenesis of this bacterium. Wang at al. [67] identified a novel SP1 protein which is secreted by $S$. suis type 2 and interacts with the host's peptidoglycan recognition protein (PGLYRP-1), inhibiting the PGLYRP1-mediated bacteriostatic effect. Wan et al. [68] described a novel SntA protein localized in the cell wall of S. suis type 2 that binds to heme, thus acquiring iron for the bacterium from the host's haemoglobin and other haemoproteins. Moreover, SntA protein can interact with the host antioxidant protein and inhibit its activity. Zhang et al. [69] identified a novel HP1330 pro-inflammatory protein that contributes markedly to $S$. suis type 2 virulence by inducing TLR2- and extracellular signal-regulated kinase 1/2 (ERK1/2)-dependent robust cytokine production, and by increasing bacterial loads in vivo, implying that HP1330 may be associated with STSLS caused by $S$. suis.

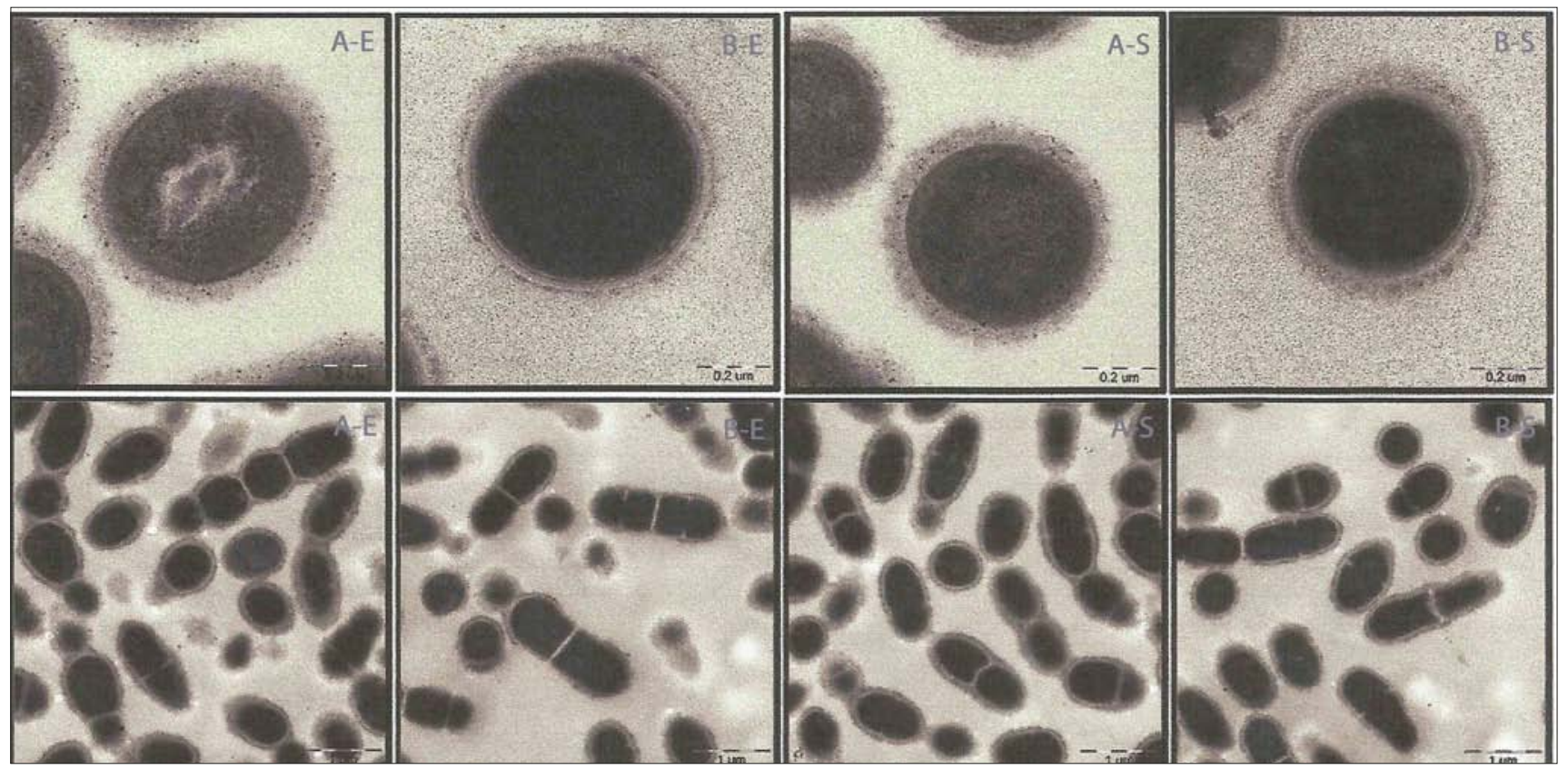

Figure 3. Transmission electron micrographs of $S$. suis strains: wild strain (A) and a codY-mutated strain (B) in the exponential (A-E versus B-E), and stationary (A-S versus $B-S$ ) phases. Bars in upper (magnification $\times 25,000$ ) and lower panels (magnification $\times 5,000$ ) are $200 \mathrm{~nm}$ and $1,000 \mathrm{~nm}$, respectively. Statistical analysis revealed that the thickness of capsules for the wild type (WT) was significantly greater compared to mutant strain, more for exponential $(P<0.0001)$ than for stationary phase $(P<0.05)$. According to Feng et al. [58] 

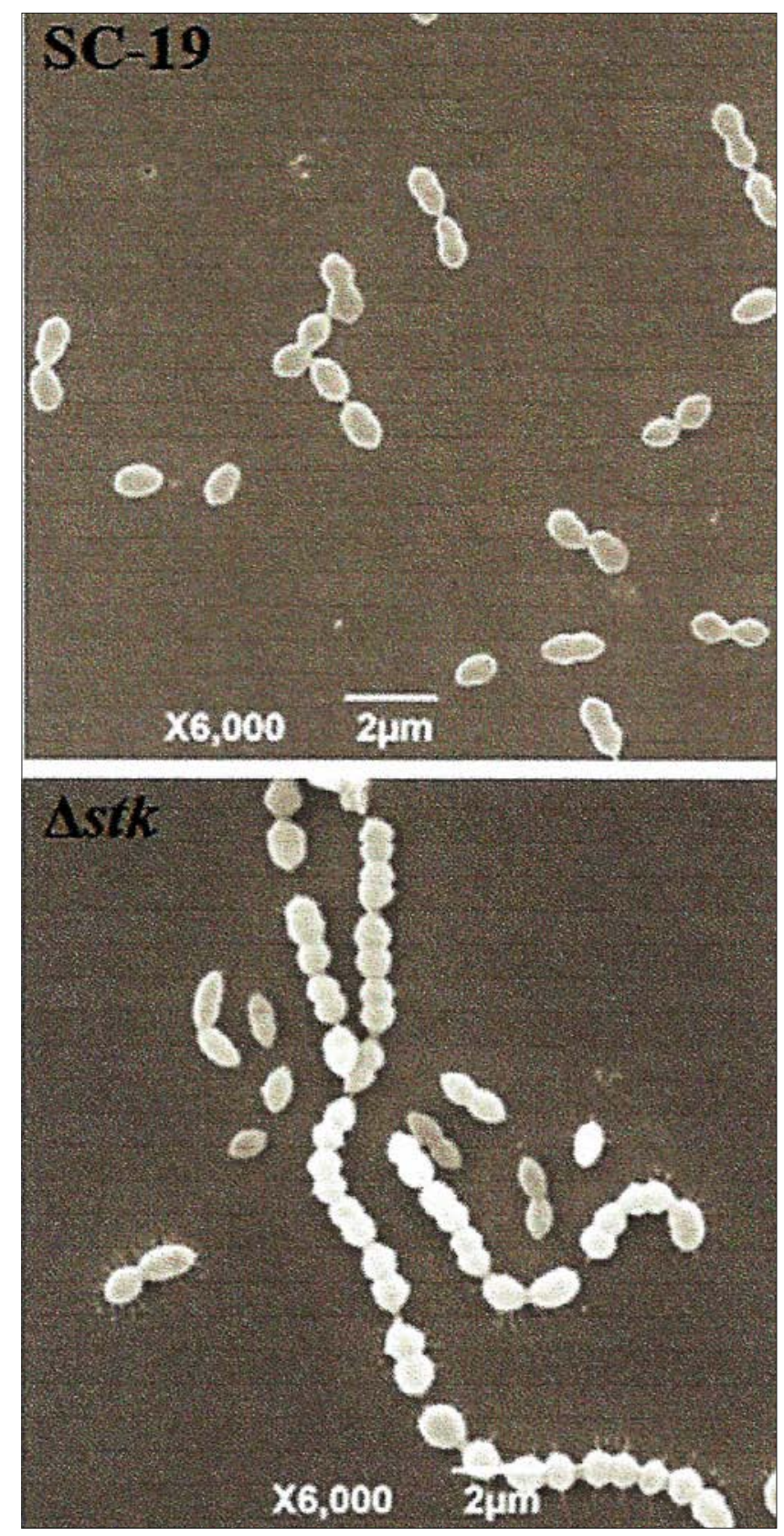

Figure 4. Scanning electron micrographs of the S. suis strains: SC-19 parent strain having serine/threonine kinase coding gene (stk) (above), and $\Delta$ stk strain with deleted stk gene (below). The mutant strain $\Delta$ stk showed significantly increased chain sizes compared with those of the parent strain SC-19. According to Zhang et al. [63]

\section{GENOMIC DETERMINANTS OF PATHOGENICITY}

The most significant discovery in the study of genomic determinants of $S$. suis pathogenicity was that by Chen et al. [70] in 2007, who detected in the genomes of 2 S. suis type 2 strains isolated from fatal human cases with STSLS diagnosis during the Chinese epidemics in Jiangsu (1998) and Sichuan (2005), the presence of a novel DNA segment of $89 \mathrm{~kb}$ in length with evident pathogenic traits and named it $89 \mathrm{~K}$ Pathogenicity Island (89K PAI). This was the first description of the genomic virulence factor in S. suis corresponding to the definition of Pathogenicity Island (PAI), which had already been described in other species of bacteria as a distinct type of genomic island (GI) that plays an important role in the rapid adaptation and increased virulence of pathogens $[70,71]$.

Further research performed during the next 10 years revealed that $89 \mathrm{~K}$ PAI comprises genes encoding a number of novel virulence factors, such type IV-like secretion system (T4SS), ABC-type export systems, SalK-SalR 2-compartment signal transduction system, and haemolysin-III-related protein (Hhly3), which are significant in the etiopathogenesis of STSLS [4, 72-74]. Zheng et al. [73] estimated that more than one-third of the hypothetical virulence factors were found to be associated with the pathogenicity island termed 89K PAI.

Li et al. [71] demonstrated that $89 \mathrm{~K}$ PAI can be acquired by horizontal transfer. They provided evidence that an $89 \mathrm{~K}$-borne integrase mediates the site specific excision and circularization of $89 \mathrm{~K}$ PAI, with contribution from an auxiliary protein named excisionase. The $89 \mathrm{~K}$ extrachromosomal circular excision product is self-transmissible and can conjugally transfer via a plasmid-like process into $S$. suis recipient cells through the $89 \mathrm{~K}$ encoded GI-type T4SS transport conduit, at a frequency of $10^{-6}$ transconjugants per donor. This was the first description of a horizontal transfer of a PAI mediated by a GI-type T4SS in a Gram-positive bacterium [71].

The extensive horizontal gene transfer and recombination observed within the populations of S. suis foster an evolution of this species which probably aims towards increased pathogenicity $[10,19,75,76]$. Okura et al. [43] stated that the marked variation of $S$. suis CPS is generated, at least in part, by the accumulation of intraspecies cps gene transfers and spontaneous mutations. More recently, Okura et al. [77] confirmed the view that $S$. suis has an open pan-genome characterized by a frequent gene transfer and a large diversity. This diversity was manifested, among others, by the presence of a novel locus encoding variable defence systems against invading DNA, discovered for the first time in bacteria. Large genomic diversity among $S$. suis strains has been confirmed by Baig et al. [78]. These authors described a clade of 9 divergent $S$. suis isolates, which were isolated from pigs in England and showed clinical and phenotypic properties of S. suis, but revealed a considerable diversity in their core and accessory genomes when compared to most $S$. suis isolates.

Ye et al. [19] performed genomic comparisons of 3 S. suis strains belonging to 3 sequence types (ST): intermediately pathogenic (ST25), highly pathogenic (ST1), and epidemic (ST7) that caused the Chinese epidemics with a high fatality and contains $89 \mathrm{~K}$ PAI. The authors demonstrated that compared to ST25 strain, the ST1 strain had acquired 132 genomic islands, including 5 pathogenicity islands containing genes encoding virulence factors, such as suilysin, extracellular protein factor, superoxide dismutase A, extracellular serine protease and prolipoprotein signal peptidase, and that ST7, the epidemic strain, had acquired an additional 5 genomic islands. According to the authors, intermediately pathogenic strain ST25 has evolved to become a highly pathogenic strain ST1, which, in turn, has more recently evolved to become epidemic strain ST7 through a single-nucleotide change in the housekeeping gene thy $A$ [19]. Based on these and similar reports on the S. suis ST7 clone, Segura et al. [10] expressed the opinion that its extremely high virulence 'suggests that there is an evolution of this pathogen's virulence traits, leading to increased pathogenicity and/or better cross-species adaptation'. Although this view is certainly correct, an inverse tendency could sometimes be observed. Thus, Shi et al. [79] stated the $89 \mathrm{~K}$ PAI was lost in 9 out of $10 \mathrm{~S}$. suis epidemic 
strains isolated between 2008-2015 from the Chinese patients with meningitis. Nevertheless, the authors stated that the loss of $89 \mathrm{~K}$ PAI might be associated with a health risk from an emerging $S$. suis type 2 population that is undergoing an evolutionary adaptation to some selection pressure from the environment, host restriction, or both.

The evolution of Streptococcus suis and emergence of virulent clones was elucidated by the authors who used the Bayesian evolutionary method for the construction of phylogenetic trees, showing the history of the species in past decades or centuries. The method was originally developed more than 250 years ago by the English statistician and philosopher Thomas Bayes (1701-1761) as a theorem for computing the posterior probability, and nowadays adopted for phylogenetic inference based on genetic data [80, 81]. Using this method, Weinert et al. [75] analysed genomes of 375 isolates with detailed clinical phenotypes from pigs and humans from the UK and Vietnam and identified 5 distinct populations, of which population number one harboured all virulent (zoonotic) human isolates and many virulent pig isolates. By dated phylogeny, the authors estimated the origin of this virulent group to be 1921. This coincides with the wide-scale introduction of indoor rearing of meat-producing pigs in larger groups which foster a recombination level, according to the authors very high among S. suis strains, and emerging of virulent clones. Among pig isolates, the authors found significant genetic differences between isolates causing 'systemic' illness (of brain, blood and joints), isolates causing respiratory illness and non-clinical isolates. The diseasecausing isolates had smaller genomes. Systemic isolates had around 50 genes fewer than respiratory isolates, and these had around 50 genes fewer than the non-clinical isolates. By contrast, genes encoding known or putative virulence factors were over-represented in systemic isolates.

The work of Weinert et al. [75] has been further developed by Willemse et al. [76] who compared, also with the use of Bayesian analysis, whole genomes of 98 S. suis isolates from human patients and pigs with invasive disease in the Netherlands, and validated their observations with 18 complete and publicly available sequences. The authors identified 7 populations, of which 2 were zoonotic. They observed, similarly as Weinert et al. [75], that zoonotic isolates had smaller genomes than non-zoonotic isolates, but contained more virulence factors. Genes that contributed the most to this difference encoded for 7 virulence factors, including a capsular polysaccharide biosynthesis gene (cps2F), an $\mathrm{N}$-acetylneuraminic acid synthetase (neuB), an extracellular protein factor (epf), a Rgg-like transcriptional regulator, an $\mathrm{ABC}$-type multidrug transport system, suilysin $(s l y)$, and endo- $\beta$-N-acetylglucosaminidase D. The authors identified a zoonotic $S$. suis clone that diverged from a nonzoonotic clone by means of gene loss, a capsule switch, and acquisition of a 2-component signalling system which took place, according to the authors, in the late 19th century (several decades earlier compared to the estimate made by Weinert et al. [75]), when foreign pig breeds were introduced into the Netherlands enabling a gene exchange between S. suis strains carried by indigenous and imported pigs. Summarizing, the results of Willemse et al. [76] indicate that the zoonotic potential of $S$. suis results from gene loss, recombination and horizontal gene transfer events. According to the authors, this potential is determined rather by accessory than core genome.
Du et al. [82] recently used the Bayesian method for uncovering events preceding the 2 largest ever epidemics caused by S. suis among the rural populations of the Chinese provinces Jiangsu and Sichuan in 1998-2005, respectively. The authors analyzed whole genomes of 95 outbreak-associated isolates and classified them into 6 clades. Phylogenetic analysis revealed that clade 1, responsible for the 1998 outbreak, emerged in October 1997, whereas clades 2-6, responsible for the 2005 outbreak, emerged separately during February 2002 - August 2004. By the end of 2004, a total of 41 lineages of $S$. suis emerged and rapidly expanded to 68 genome types through single base mutations when the outbreak occurred in June 2005. The origin of the Jiangsu and Sichuan S. suis ST7 strains was common and dated back to 1996. Analysis of the Sichuan outbreak showed that during the epidemic, highly virulent strains emerged over a large area and persons were infected in parallel in distant geographic locations. Based on these results, the authors established that the piglets were infected with an extremely virulent $S$. suis ST7 strain in the industrialized piglet breeding companies before distribution to the backyard farmers across wide geographic regions. These companies were established to increase pork production by replacement of local pig breeds in China with the more efficient imported breeds. Piglets of imported breeds were produced through large companies and distributed to backyard farmers who raised them, often in poor hygienic conditions. Such a combination of a highly industrialized piglet supply system and the farmer's backyard animal raising practices resulted in the creation of a high risk of outbreaks of infectious disease on an unprecedented scale, and appeared to be an unforeseen consequence of economic development. The analysis performed by Du et al. [82] proved to be very helpful for the delineation of the future prevention strategies that should include disease monitoring at the breeding companies, a pathogen-free supply of piglets and improved hygiene for backyard farmers.

\section{IMPORTANCE OF BIOFILM FORMATION}

Biofilms are highly structured microbial communities embedded in a self-generated organic polymer matrix and adhere to abiotic or biological surfaces. The matrix protects microbes against the innate and adaptive host defence mechanisms, treatment with antibiotics and unfavourable environmental conditions, such as desiccation, nutritional or oxidative stress, and UV light exposure. Thus, the formation of biofilms increases the persistence of bacteria, both in the hosts and the external environment. The presence of biofilms in biomaterials used in surgery (e.g. vascular catheters, prosthetic joints and artificial heart valves) is one of the main causes of difficult-to-cure infections [3, 6, 61, 83, 84].

The first report on biofilm production by a $S$. suis was presented by Bonifait et al. [85] who found that incorporation of fibrinogen during growth induced the formation of a thick biofilm resulting in antibiotic resistance. The increased resistance of S. suis in biofilms to penicillin G and ampicillin was confirmed by Grenier et al. [86]. Tanabe et al. [87] demonstrated that inactivation of CPS expression in S. suis results in a significant increase in cell surface hydrophobicity combined with a capacity to form biofilms (Fig. 5). This suggests that the CPS may hinder hydrophobic molecules that mediate biofilm formation $[6,87]$. 


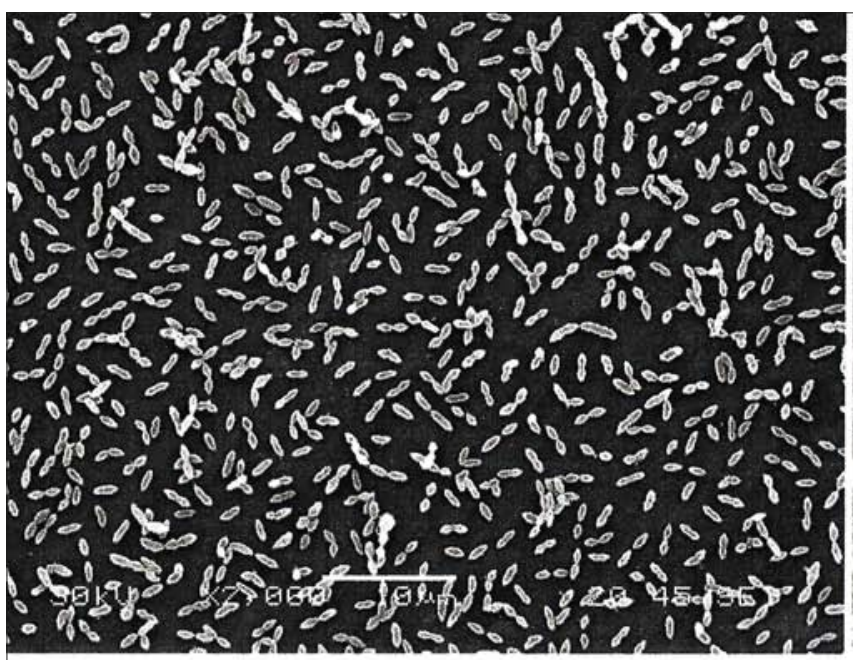

$\mathbf{S 7 3 5}$

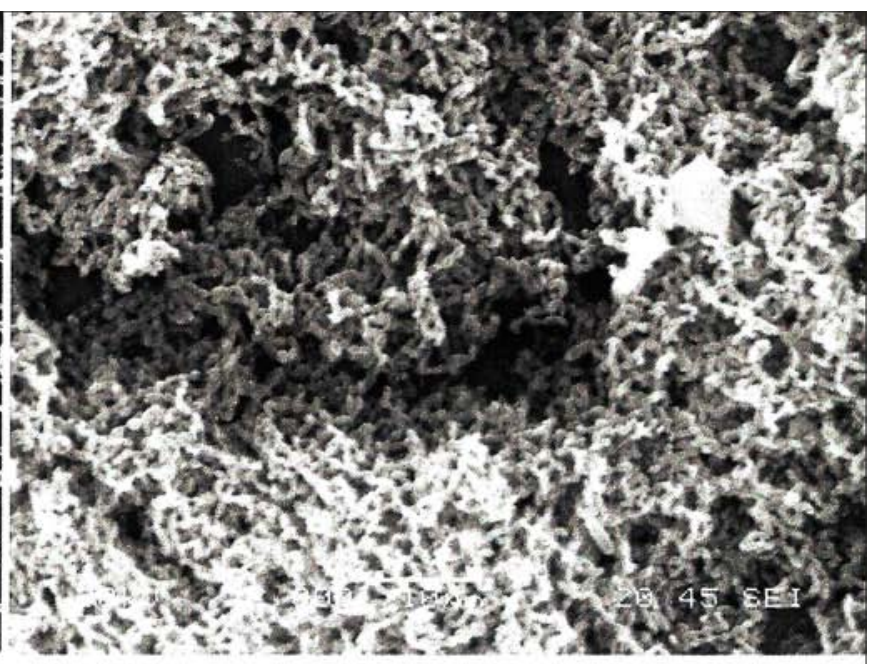

BD101

Figure 5. Scanning electron micrographs of S. suis biofilms formed after $24 \mathrm{~h}$ of growth by the Streptococcus suis strain S735 and its unencapsulated mutant BD101. Loss of capsule significantly enhanced the biofilm formation. According to Tanabe et al. [87], with permission

Dawei et al. [83] studied biofilm-forming potential of 46 S. suis strains isolated from humans and pigs in China. They detected in a majority of strains, already in one-daycultures, massive amounts of mucus-like extracellular polysaccharides (exopolysaccharides) surrounding bacterial cells and forming a biofilms matrix. The exopolysaccharides were visualized by green fluorescence when stained with fluorescein isothiocyanate-concanavalin A (FITC-ConA) and observed with electron microscope (Fig. 6). S. suis produced biofilms both on smooth and rough surfaces; however, the production was more abundant on a rough surface of organic membrane than on the smooth surface of a glass coverslip (Fig. 7). The nutritional contents including glucose and $\mathrm{NaCl}$ in the growth medium modulated biofilm formation, with the optimal concentrations equal to $1 \%$ for glucose and $0.5 \%$ for $\mathrm{NaCl}$. The authors stated a significant, type-dependent differences in biofilm-forming potential

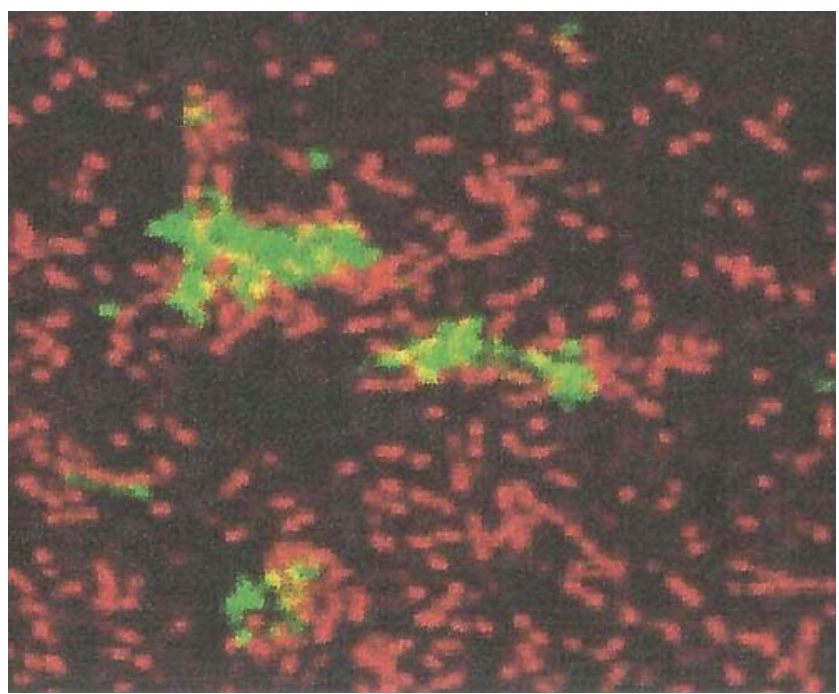

Figure 6. Confocal laser scanning (CLS) image of S. suis NJ-3 biofilm on glass coverslip. The extracellular polysaccharide in the biofilm was visualized with fluorescein isothiocyanate - concanavalin A (FITC-ConA) which fluoresces green. The bacterial cells in biofilms were visualized by staining with propidium iodide (PI) which fluoresces red. According to Dawei et al. [83], modified among the tested strains. Overall, the ability to produce biofilm was significantly greater in type 9 strains than in strains belonging to other types. However, 2 S. suis type 2 strains isolated from human patients showed the strongest biofilm forming abilities. Biofilm formation was inhibited by low concentrations of 5 commonly used antimicrobial agents: penicillin, erythromycin, azithromycin, ciprofloxacin, and ofloxacin [83].

Wang et al. [88] found that 2 virulent S. suis type 2 strains displayed increased biofilm formation compared to an avirulent one. Bacteria in biofilms showed a reduced virulence, which, according to authors, could be helpful for bacteria in the achievement of persistent infection in vivo by keeping a host alive. Meng et al. [89] demonstrated that 10 out of the 32 examined S. suis strains showed the biofilm-forming ability, and in this state exhibited increased resistance to a number of antibiotics. The authors demonstrated that the bacteriophage lysine caused a significant reduction of S. suis biofilms and acted synergistically in combination with antibiotics, thus showing a new possibilities for an effective treatment of S. suis infections. Recently, Bai et al. [84] found that the application of the aqueous extract of the leaves of Syringa oblata, an Oleaceae plant which proved to have the most developmental potential of Chinese Medicine, might be used for the control of biofilm formation by $S$. suis. The authors found that this extract affected growth, metabolism and the CPS synthesis enzymes. The active ingredient of the Syringa oblata extract was rutin, a citrus flavonoid glycoside present in many foods, having anti-inflammatory, antimicrobial, antioxidant, and antihypertensive effects. Wang et al. [90] demonstrated that rutin inhibits $S$. suis biofilm formation by affecting CPS biosynthesis and bacterial adhesion (Fig. 8). In parallel, Ding et al. [91] found that $S$. suis biofilm formation could be inhibited by rhubarb water extract, another Chinese herbal medication.

Wang et al. $[92,93]$ also demonstrated that the formation of biofilm by $S$. suis is dependent on the action of the LuxS (S-ribosyl homocysteinase) enzyme, already mentioned in this article at the reviewing of enzymatic virulence factors. LuxS catalyzes synthesis of auto-inducer 2 (AI-2), a signal 

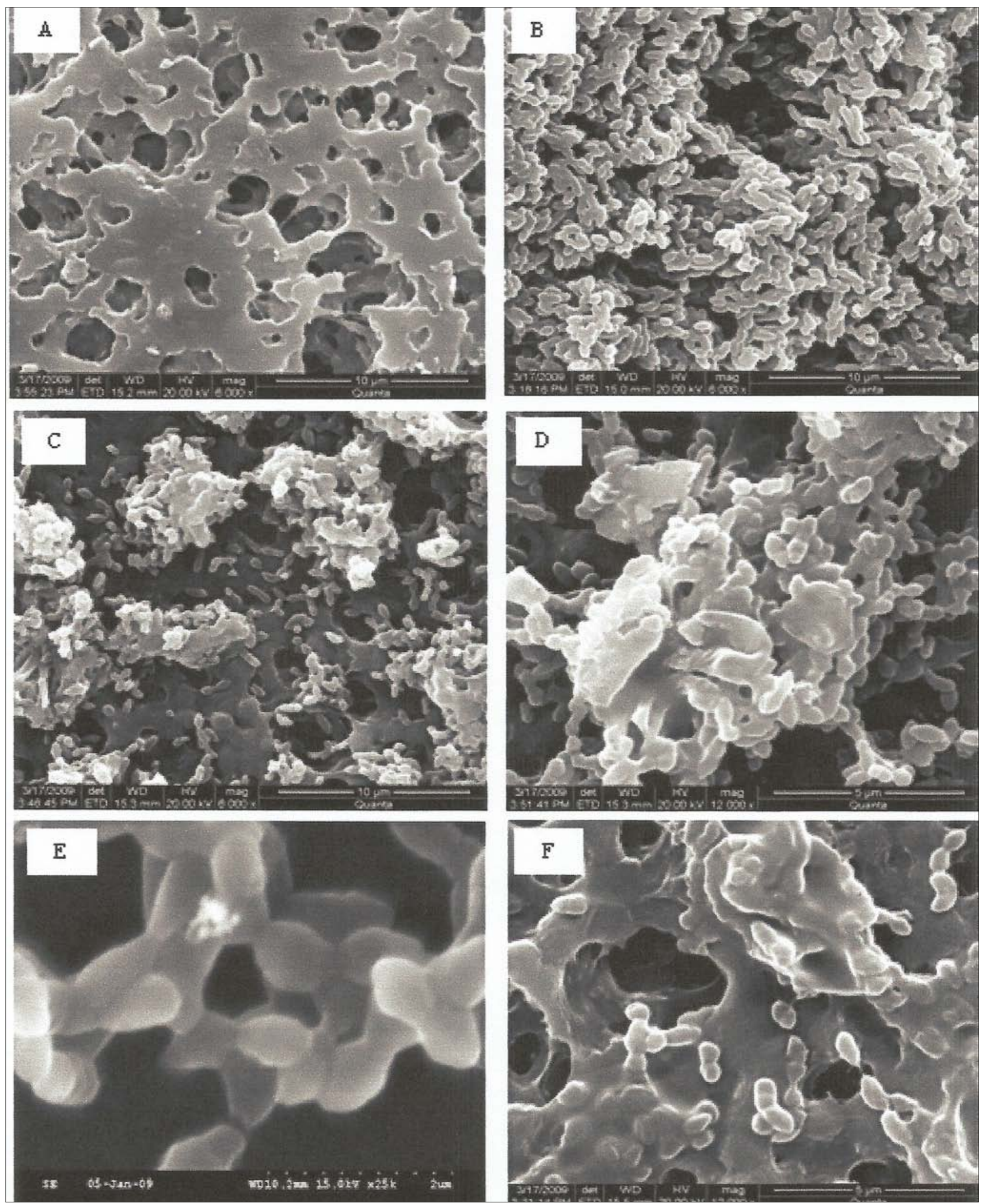

Figure 7. Scanning electron micrographs of the biofilms formed by S. suis NJ-3 and YY060816 strains on rough organic membrane and smooth glass coverslip. Bacteria were grown in 6-well tissue culture plates with organic membranes and glass coverslips at $37^{\circ} \mathrm{C}$ for $24 \mathrm{~h}$. Massive amounts of mucus-like extracellular materials were observed. A: Rough organic membrane; B: S. suis NJ-3 planktonic cells; C: Biofilm of S. suis NJ-3 on rough organic membrane ( $\times 6,000)$; D: Biofilm of S. suis NJ-3 on rough organic membrane $(\times 12,000)$; E: Biofilm of S. suis NJ-3 on smooth glass coverslip $(\times 25,000)$; F: Biofilm of S. suis YY060816 on rough organic membrane. According to Dawei et al. [83] 

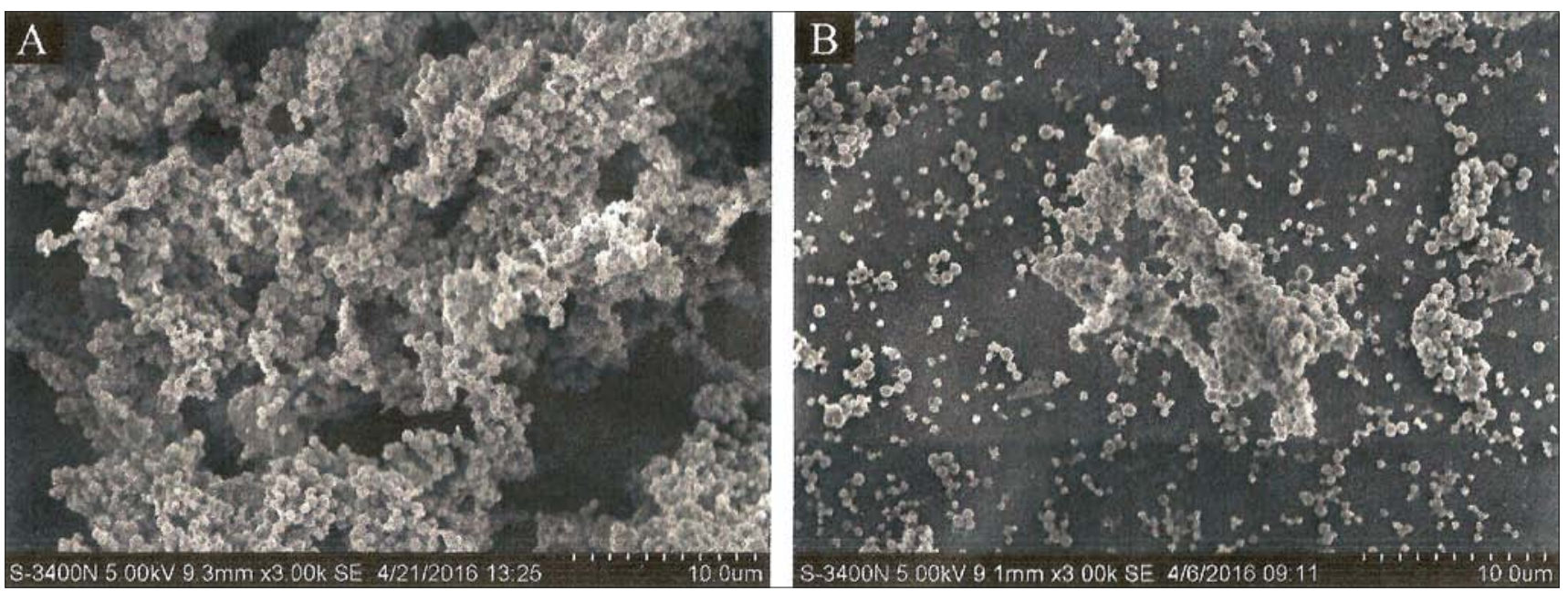

Figure 8. Effect of rutin on S. suis biofilm visualized by scanning electron microscope. A: Biofilm formation of S. suis without rutin; B: Biofilm formation of S. suis with $1 / 4$ MIC $(0.0781 \mathrm{mg} / \mathrm{ml})$ rutin treatment. According to Wang et al. [90]

molecule utilized in intra- and interspecies quorum sensing (QS) communication system regulating the life functions of bacteria. Decreased levels of S. suis biofilm formation and host-cell adherence, as well as the inability to produce AI-2, were observed in mutants with deleted luxS gene. In contrast, exogenous addition of a low concentration of AI-2 synthesized in vitro was found to promote biofilm formation and host-cell adherence [92]. The authors showed that the catalytically active LuxS exists in S. suis as a homodimer in solution and identified 2 key amino acids: Phe80 and His87, which are located near the substrate binding site and regulate the AI-2 production and biofilm formation in S. suis [93].

Interesting results were obtained recently by Ma et al. [94] who demonstrated experimentally that $S$. suis type 2 can form biofilms in vivo in some murine tissues, such as the liver, spleen and kidney. The bacteria in the biofilms were not phagocytized. Biofilm formation increased up to 6-fold in the presence of neutrophils and the biofilm extracellular matrix can inhibit release of the neutrophil extracellular traps (NETs) and increase the survival of bacteria in the blood. Nevertheless, the protection is not complete and according to the authors NETs appear to be an important mechanism to eliminate $S$. suis biofilms [94].

\section{CONCLUSION}

Based on the presented review, it is evident that Streptococcus suis has an incredible ability to adapt to the changing environmental conditions which enables the bacterium to evade the host's immune defence and successfully colonize its tissues. The evolution of this zoonotic organism tends most probably towards a greater virulence creating a growing risk for humans and animals. For the protection of the millions of humans who have an occupational contact with pigs and pork, and to avoid huge economic losses every year due to disease in pigs, the best prevention measure would be a vaccine equally efficient for humans and pigs. The task is not easy because of the great diversity among S. suis strains. Nevertheless, the research aimed at obtaining such vaccine is being undertaken by many prominent scientists worldwide, some of whom have recently published promising results in this area $[55,95,96,97]$. We appeal to the governmental and international organizations, such as UNESCO, WHO, FAO, the European Union, and others, for a significant financial support of such research that would hopefully result in obtaining an effective, universal and licensed vaccine within the next 5-10 years.

\section{Acknowledgements}

The authors express their thanks to the Veterinary Medical Association in Ottawa, Canada, for kind permission to reproduce Figure 2 from the article by Roy et al. [18] and Figure 5 from the article by Tanabe et al. [87], both published in the Canadian Journal of Veterinary Research in 2015 and 2010, respectively. We are also grateful to the editors of the following journals, Veterinary Research, Frontiers in Cellular and Infection Microbiology, Scientific Reports and the Brazilian Journal of Microbiology who granted permission fo the reproduction of Figure 1 from the article by Lecours et al. [23], Figure 3 from the article by Feng et al. [58], Figure 4 from the article by Zhang et al. [63], Figures 6 and 7 from the article by Dawei et al. [83], and Figure 8 from the article by Wang et al. [90], respectively, under the terms of the Creative Commons Attribution License.

\section{REFERENCES}

1. Gottschalk M, Xu J, Calzas C, Segura M. Streptococcus suis: a new emerging or an old neglected zoonotic pathogen? Future Microbiol. 2010; 5(3): 371-391.

2. Fittipaldi N, Segura M, Grenier D, Gottschalk M. Virulence factors involved in the pathogenesis of the infection caused by the swine pathogen and zoonotic agent Streptococcus suis. Future Microbiol. 2012; 7(2): 259-79.

3. Fulde M, Valentin-Weigand P. Epidemiology and pathogenicity of zoonotic streptococci. Curr Top Microbiol Immunol. 2013; 368: 49-81.

4. Feng Y, Zhang H, Wu Z, Wang S, Cao M, Hu D, Wang C. Streptococcus suis infection: an emerging/reemerging challenge of bacterial infectious diseases? Virulence. 2014; 5(4): 477-497.

5. Dutkiewicz J, Sroka J, Zając V, Wasiński B, Cisak E, Sawczyn A, Kloc A, Wójcik-Fatla A. Streptococcus suis: a re-emerging pathogen associated with occupational exposure to pigs or pork products. Part I. Epidemiology. Ann Agric Environ Med. 2017; 24(4): 683-695.

6. Segura M, Calzas C, Grenier D, Gottschalk M. Initial steps of the pathogenesis of the infection caused by Streptococcus suis: fighting against nonspecific defenses. FEBS Lett. 2016; 590(21): 3772-3799. 
7. Kouki A, Pieters RJ, Nilsson UJ, Loimaranta V, Finne J, Haataja S. Bacterial adhesion of Streptococcus suis to host cells and its inhibition by carbohydrate ligands. Biology (Basel). 2013; 2(3): 918-935.

8. Auger JP, Gottschalk M. The Streptococcus suis factor H-binding protein: A key to unlocking the blood-brain barrier and access the central nervous system? Virulence. 2017; 8 (7): 1081-1084, doi: $10.1080 / 21505594.2017 .1342027$

9. Ferrando ML, de Greeff A, van Rooijen WJ, Stockhofe-Zurwieden N, Nielsen J, Wichgers Schreur PJ, Pannekoek Y, Heuvelink A, van der Ende A, Smith H, Schultsz C. Host-pathogen interaction at the intestinal mucosa correlates with zoonotic potential of Streptococcus suis. J Infect Dis. 2015 ; 212(1): 95-105.

10. Segura M, Fittipaldi N, Calzas C, Gottschalk M. Critical Streptococcus suis virulence factors: are they all really critical? Trends Microbiol. 2017; 25(7): 585-599.

11. Gottschalk M, Segura M, Xu J. Streptococcus suis infections in humans: the Chinese experience and the situation in North America. Anim Health Res Rev. 2007; 8(1): 29-45.

12. Liu H, Zhu S, Sun Y, Li N, Gu J, Sun C, Feng X, Han W, Jiang JX, Lei L. Selection of potential virulence factors contributing to Streptococcus suis Type 2 penetration into the Blood Brain Barrier in an in vitro coculture model. J Microbiol Biotechnol. 2017; 27(1): 161-170.

13. Kong D, Chen Z, Wang J, Lv Q, Jiang H, Zheng Y, Xu M, Zhou X, Hao $\mathrm{H}$, Jiang $\mathrm{Y}$. Interaction of factor H-binding protein of Streptococcus suis with globotriaosylceramide promotes the development of meningitis. Virulence 2017; 8(7): 1290-1302.

14. Strangmann E, Fröleke H, Kohse KP. Septic shock caused by Streptococcus suis: case report and investigation of a risk group. Int J Hyg Environ Health. 2002; 205(5): 385-392.

15. Domínguez-Punaro MC, Koedel U, Hoegen T, Demel C, Klein M, Gottschalk M. Severe cochlear inflammation and vestibular syndrome in an experimental model of Streptococcus suis infection in mice. Eur J Clin Microbiol Infect Dis. 2012; 31(9): 2391-2400.

16. Segura M. Fisher scientific award lecture - the capsular polysaccharides of Group B Streptococcus and Streptococcus suis differently modulate bacterial interactions with dendritic cells. Can J Microbiol. 2012; 58(3): 249-260.

17. Houde M, Gottschalk M, Gagnon F, Van Calsteren MR, Segura M. Streptococcus suis capsular polysaccharide inhibits phagocytosis through destabilization of lipid microdomains and prevents lactosylceramide-dependent recognition. Infect Immun. 2012; 80(2): 506-517.

18. Roy D, Auger JP, Segura M, Fittipaldi N, Takamatsu D, Okura M, Gottschalk M. Role of the capsular polysaccharide as a virulence factor for Streptococcus suis serotype 14. Can J Vet Res. 2015; 79(2): 141-146.

19. Ye C, Zheng H, Zhang J, Jing H, Wang L, Xiong Y, Wang W, Zhou Z, Sun Q, Luo X, Du H, Gottschalk M, Xu J. Clinical, experimental, and genomic differences between intermediately pathogenic, highly pathogenic, and epidemic Streptococcus suis. J Infect Dis. 2009; 199(1): 97-107.

20. De Buhr N, Neumann A, Jerjomiceva N, von Köckritz-Blickwede M, Baums CG. Streptococcus suis DNase SsnA contributes to degradation of neutrophils extracellular traps (NETs) and evasion of NET-mediated antimicrobial activity. Microbiology. 2014; 160(2): 385-395.

21. De Buhr N, Stehr M, Neumann A, Naim HY, Valentin-Weigand P, von Köckritz-Blickwede M, Baums CG. Identification of a novel DNase of Streptococcus suis (EndAsuis) important for neutrophil extracellular trap degradation during exponential growth. Microbiology. 2015; 161(4): 838-850

22. De Buhr N, Reuner F, Neumann A, Stump-Guthier C, Tenenbaum T, Schroten H, Ishikawa H, Müller K, Beineke A, Hennig-Pauka I, Gutsmann T, Valentin-Weigand P, Baums CG(, von Köckritz-Blickwede M. Neutrophil extracellular trap formation in the Streptococcus suisinfected cerebrospinal fluid compartment. Cell Microbiol. 2017; 19(2). doi: $10.1111 / \mathrm{cmi} .12649$.

23. Lecours MP, Segura M, Lachance C, Mussa T, Surprenant C, Montoya M, Gottschalk M. Characterization of porcine dendritic cell response to Streptococcus suis. Vet Res. 2011; 42:72. doi: 10.1186/1297-9716-42-72.

24. Merad M, Sathe P, Helft J, Miller J, Mortha A. The dendritic cell lineage: ontogeny and function of dendritic cells and their subsets in the steady state and the inflamed setting. Annu Rev Immunol. 2013; 31. doi:10.1146/annurev-immunol-020711-074950.

25. Lecours MP, Segura M, Fittipaldi N, Rivest S, Gottschalk M. Immune receptors involved in Streptococcus suis recognition by dendritic cells. PloS One. 2012; 7(9): e44746. doi: 10.1371/journal.pone.0044746.

26. Lecours MP, Letendre C, Clarke D, Lemire P, Galbas T, BenoitBiancamano MO, Thibodeau J, Gottschalk M, Segura M. Immune- responsiveness of $\mathrm{CD}^{+} \mathrm{T}$ cells during Streptococcus suis serotype 2 infection. Sci Rep. 2016; 6: 38061, doi: 10.1038/srep38061.

27. Lemire P, Galbas T, Thibodeau J, Segura M. Natural Killer Cell functions during the innate immune response to pathogenic streptococci. Front Microbiol. 2017; 8: 1196. doi: 10.3389/fmicb.2017.01196.

28. Martelet L, Lacouture S, Goyette-Desjardins G, Beauchamp G, Surprenant C, Gottschalk M, Segura M. Porcine dendritic cells as an in vitro model to assess the immunological behaviour of Streptococcus suis subunit vaccine formulations and the polarizing effect of adjuvants. Pathogens. 2017; 6, 13. doi: 10.3390/pathogens6010013

29. Perry VH, Teeling J. Microglia and macrophages of the central nervous system: the contribution of microglia priming and systemic inflammation to chronic neurodegeneration. Semin Immunopathol. 2013; 35: 601-612.

30. Sofroniew MV, Vinters HV. Astrocytes: biology and pathology. Acta Neuropathol. 2010; 119: 7-35.

31. Domínguez-Punaro MC, Segura M, Plante MM, Lacouture S, Rivest S, Gottschalk M. Streptococcus suis serotype 2, an important swine and human pathogen, induces strong systemic and cerebral inflammatory responses in a mouse model of infection. J Immunol. 2007; 179(3): 1842-1854.

32. Domínguez-Punaro ML, Segura M, Contreras I, Lachance C, Houde M, Lecours MP, Olivier M, Gottschalk M. In vitro characterization of the microglial inflammatory response to Streptococcus suis, an important emerging zoonotic agent of meningitis. Infect Immun. 2010; 78(12): 5074-5085.

33. Zheng H, Sun H, Dominguez-Punaro ML, Bai X, Ji S, Segura M, Xu J. Evaluation of the pathogenesis of meningitis caused by Streptococcus suis sequence type 7 using the infection of BV2 microglial cells. J Med Microbiol. 2013; 62: 360-368.

34. Seele J, Nau R, Prajeeth CK, Stangel M, Valentin-Weigand P, Seitz M. Astrocytes enhance Streptococcus suis-glial cell interaction in primary astrocyte-microglial cell co-cultures. Pathogens. 2016; 5: 43. doi: 10.3390/pathogens5020043.

35. Zhao Y, Liu G, Li S, Wang M, Song J, Wang J, Tang J, Li M, Hu F. Role of a type IV-like secretion system of Streptococcus suis 2 in the development of streptococcal toxic shock syndrome. J Infect Dis. 2011; 204(2): 274-281.

36. Jiang X, Yang Y, Zhou J, Zhu L, Gu Y, Zhang X, Li X, Fang W. Roles of the putative type IV-like secretion system key component VirD4 and PrsA in pathogenesis of Streptococcus suis Type 2. Front Cell Infect Microbiol. 2016; 6:172. doi: 10.3389/fcimb.2016.00172

37. Charland N, Harel J, Kobisch M, Lacasse S, Gottschalk M. Streptococcus suis serotype 2 mutants deficient in capsular expression. Microbiology 1998; 144: 325-332.

38. Smith HE, Damman M, van der Velde J, Wagenaar F, Wisselink HJ, Stockhofe-Zurwieden N, Smits MA. Identification and characterization of the cps locus of Streptococcus suis serotype 2: the capsule protects against phagocytosis and is an important virulence factor. Infect Immun. 1999; 67(4): 1750-1756.

39. Vecht U, Wisselink HJ, Jellema ML, Smith HE. Identification of two proteins associated with virulence of Streptococcus suis type 2. Infect Immun. 1991; 59(9): 3156-3162.

40. Jacobs AAC, Loeffen PLW, van den Berg AJG, Storm PK. Identification, purification, and characterization of a thiol-activated hemolysin (suilysin) of Streptococcus suis. Infect Immun. 1994; 62: 1742-1748.

41. Gottschalk MG, Lacouture S, Dubreuil JD. Characterization of Streptococcus suis capsular type 2 haemolysin. Microbiology. 1995; 141: 189-195.

42. Gomez E, Kennedy CC, Gottschalk M, Cunningham SA, Patel R, Virk A. Streptococcus suis-related prosthetic joint infection and streptococcal toxic shock-like syndrome in a pig farmer in the United States. J Clin Microbiol. 2014; 52(6): 2254-2258.

43. Okura M, Takamatsu D, Maruyama F, Nozawa T, Nakagawa I, Osaki M, Sekizaki T, Gottschalk M, Kumagai Y, Hamada S. Genetic analysis of capsular polysaccharide synthesis gene clusters from all serotypes of Streptococcus suis: potential mechanisms for generation of capsular variation. Appl Environ Microbiol. 2013; 79(8): 2796-2806.

44. Lakkitjaroen N, Takamatsu D, Okura M, Sato M, Osaki M, Sekizaki T. Capsule loss or death: the position of mutations among capsule genes sways the destiny of Streptococcus suis. FEMS Microbiol Lett. 2014; 354(1): 46-54.

45. Calzas C, Goyette-Desjardins G, Lemire P, Gagnon F, Lachance C, Van Calsteren MR, Segura M. Group B Streptococcus and Streptococcus suis capsular polysaccharides induce chemokine production by dendritic cells via Toll-like receptor 2- and MyD88-dependent and -independent pathways. Infect Immun. 2013; 81(9): 3106-3118. 
46. Calzas C, Lemire P, Auray G, Gerdts V, Gottschalk M, Segura M. Antibody response specific to the capsular polysaccharide is impaired in Streptococcus suis serotype 2-infected animals. Infect Immun. 2015; 83(1): 441-453.

47. Calzas C, Taillardet M, Fourati IS, Roy D, Gottschalk M, Soudeyns H, Defrance T, Segura M. Evaluation of the immunomodulatory properties of Streptococcus suis and Group B Streptococcus capsular polysaccharides on the humoral response. Pathogens. 2017; 6(2). Pii: E16. doi: 10.3390/pathogens6020016.

48. Auger JP, Meekhanon N, Okura M, Osaki M, Gottschalk M, Sekizaki T, Takamatsu D. Streptococcus suis serotype 2 capsule in vivo. Emerg Infect Dis. 2016; 22(10): 1793-1796.

49. Tohya M, Watanabe T, Maruyama F, Arai S, Ota A, Athey TB, Fittipaldi N, Nakagawa I, Sekizaki T. Comparative genome analyses of Streptococcus suis isolates from endocarditis demonstrate persistence of dual phenotypic clones. PloS One. 2016; 11(7): e0159558. doi: 10.1371/ journal.pone.0159558.

50. Wang Y, Gagnon CA, Savard C, Music N, Srednik M, Segura M, Lachance C, Bellehumeur C, Gottschalk M. Capsular sialic acid of Streptococcus suis serotype 2 binds to swine influenza virus and enhances bacterial interactions with virus-infected tracheal epithelial cells. Infect Immun. 2013; 81(12): 4498-4508.

51. Patenge N, Fiedler T, Kreikemeyer B. Common regulators of virulence in streptococci. Curr Top Microbiol Immunol. 2013; 368: 111-153.

52. Haas B, Bonifait L, Vaillancourt K, Charette SJ, Marcelo Gottschalk M, Grenier D. Characterization of Dnase activity and gene in Streptococcus suis and evidence for a role as virulence Factor. BMC Research Notes 2014; 7:424, doi: 10.1186/1756-0500-7-424.

53. Seele J, Singpiel A, Spoerry C, von Pawel-Rammingen U, ValentinWeigand P, Baums CG. Identification of a novel host-specific IgM protease in Streptococcus suis. J Bacteriol. 2013; 195(5): 930-940.

54. Seele J, Beineke A, Hillermann LM, Jaschok-Kentner B, von PawelRammingen U, Valentin-Weigand P, Baums CG. The immunoglobulin M-degrading enzyme of Streptococcus suis, IdeSsuis, is involved in complement evasion. Vet Res. 2015; 46: 45. doi: 10.1186/s13567-0150171-6.

55. Seele J, Hillermann LM, Beineke A, Seitz M, von Pawel-Rammingen U, Valentin-Weigand P, Baums CG. The immunoglobulin M-degrading enzyme of Streptococcus suis, IdeSsuis, is a highly protective antigen against serotype 2. Vaccine. 2015; 33(19): 2207-2212.

56. Spoerry C, Seele J, Valentin-Weigand P, Baums CG, von PawelRammingen U. Identification and characterization of IgdE, a novel IgG-degrading protease of Streptococcus suis with unique specificity for porcine IgG. J Biol Chem. 2016; 291(15): 7915-7925.

57. Gao T, Tan M, Liu W, Zhang C, Zhang T, Zheng L, Zhu J, Li L, Zhou R. GidA, a tRNA modification enzyme, contributes to the growth, and virulence of Streptococcus suis serotype 2. Front Cell Infect Microbiol. 2016; 6:44. doi: 10.3389/fcimb.2016.00044.

58. Feng L, Zhu J, Chang H, Gao X, Gao C, Wei X, Yuan F, Bei W. The CodY regulator is essential for virulence in Streptococcus suis serotype 2. Sci Rep. 2016; 6:21241. doi: 10.1038/srep21241.

59. Ji X, Sun Y, Liu J, Zhu L, Guo X, Lang X, Feng S. A novel virulenceassociated protein, vapE, in Streptococcus suis serotype 2. Mol Med Rep. 2016; 13(3): 2871-2877.

60. Zhu J, Zhang T, Su Z, Li L, Wang D, Xiao R, Teng M, Tan M, Zhou R. (p) ppGpp synthetases regulate the pathogenesis of zoonotic Streptococcus suis. Microbiol Res. 2016; 191:1-11.

61. Tan MF, Liu WQ, Zhang CY, Gao T, Zheng LL, Qiu DX, Li L, Zhou R. The involvement of MsmK in pathogenesis of the Streptococcus suis serotype 2. Microbiology Open. 2017; 6(2). doi: 10.1002/mbo3.433.

62. Yuan F, Tan C, Liu Z, Yang K, Zhou D, Liu W, Duan Z, Guo R, Chen $\mathrm{H}$, Tian Y, Bei W. The $1910 \mathrm{HK} / \mathrm{RR}$ two-component system is essential for the virulence of Streptococcus suis serotype 2. Microb Pathog. 2017; 104: 137-145.

63. Zhang C, Sun W, Tan M, Dong M, Liu W, Gao T, Li L, Xu Z, Zhou R. The eukaryote-like serine/threonine kinase STK regulates the growth and metabolism of zoonotic Streptococcus suis. Front Cell Infect Microbiol. 2017; 7:66. doi: 10.3389/fcimb.2017.00066.

64. Ferrando ML, Willemse N, Zaccaria E, Pannekoek Y, van der Ende A, Schultsz C. Streptococcal Adhesin P (SadP) contributes to Streptococcus suis adhesion to the human intestinal epithelium. PloS One. 2017; 12(4): e0175639. doi: 10.1371/journal.pone.0175639.

65. Lai L, Dai J, Tang H, Zhang S, Wu C, Qiu W, Lu C, Yao H, Fan H, Wu Z. Streptococcus suis serotype 9 strain GZ0565 contains a type VII secretion system putative substrate EsxA that contributes to bacterial virulence and a vanZ-like gene that confers resistance to teicoplanin and dalbavancin in Streptococcus agalactiae. Vet Microbiol. 2017; 205: 26-33.

66. Xu J, Zheng C, Cao M, Zeng T, Zhao X, Shi G, Chen H, Bei W. The manganese efflux system MntE contributes to the virulence of Streptococcus suis serotype 2. Microb Pathog. 2017; 110: 23-30.

67. Wang J, Feng Y, Wang C, Srinivas S, Chen C, Liao H, He E, Jiang S, Tang J. Pathogenic Streptococcus strains employ novel escape strategy to inhibit bacteriostatic effect mediated by mammalian peptidoglycan recognition protein. Cell Microbiol. 2017. doi: 10.1111/cmi.12724.

68. Wan Y, Zhang S, Li L, Chen H, Zhou R. Characterization of a novel streptococcal heme-binding protein SntA and its interaction with host antioxidant protein AOP2. Microb Pathog. 2017; 111: 145-155.

69. Zhang Q, Huang J, Yu J, Xu Z, Liu L, Song Y, Sun X, Zhang A, Jin M. HP1330 contributes to Streptococcus suis virulence by inducing TollLike Receptor 2- and ERK1/2-dependent pro-inflammatory responses and influencing in vivo $S$. suis loads. Front Immunol. 2017; 8: 869. doi: 10.3389/fimmu.2017.00869.

70. Chen C, Tang J, Dong W, Wang C, Feng Y, Wang J, Zheng F, Pan X, Liu D, Li M, et al. A glimpse of streptococcal toxic shock syndrome from comparative genomics of S. suis 2 Chinese isolates. PloS One. 2007; 2(3): e315. doi: 10.1371/journal.pone.0000315

71. Li M, Shen X, Yan J, Han H, Zheng B, Liu D, Cheng H, Zhao Y, Rao X, Wang C, Tang J, Hu F, Gao GF. GI-type T4SS-mediated horizontal transfer of the $89 \mathrm{~K}$ pathogenicity island in epidemic Streptococcus suis serotype 2. Mol Microbiol. 2011; 79(6): 1670-1683.

72. Kerdsin A, Dejsirilert S, Puangpatra P, Sripakdee S, Chumla K, Boonkerd N, Polwichai P, Tanimura S, Takeuchi D, Nakayama T et al. Genotypic profile of Streptococcus suis serotype 2 and clinical features of infection in humans, Thailand. Emerg Infect Dis. 2011; 17(5): 835-842.

73. Zheng JX, Li Y, Zhang H, Fan HJ, Lu CP. Identification and characterization of a novel hemolysis-related gene in Streptococcus suis serotype 2. PloS One. 2013; 8(9): e74674. doi: 10.1371/journal. pone.0074674.

74. Segura M, Zheng H, de Greeff A, Gao GF, Grenier D, Jiang Y, Lu C, Maskell D, Oishi K, Okura M et al. Latest developments on Streptococcus suis: an emerging zoonotic pathogen: part 2. Future Microbiol. 2014; 9(5): 587-591.

75. Weinert LA, Chaudhuri RR, Wang J, Peters SE, Corander J, Jombart T, Baig A, Howell KJ, Vehkala M, Välimäki N et al. Genomic signatures of human and animal disease in the zoonotic pathogen Streptococcus suis. Nat Commun. 2015; 6:6740. doi: 10.1038/ncomms7740.|

76. Willemse N, Howell KJ, Weinert LA, Heuvelink A, Pannekoek Y, Wagenaar JA, Smith HE, van der Ende A, Schultsz C. An emerging zoonotic clone in the Netherlands provides clues to virulence and zoonotic potential of Streptococcus suis. Sci Rep. 2016; 6: 28984. doi: $10.1038 /$ srep28984.

77. Okura M, Nozawa T, Watanabe T, Murase K, Nakagawa I, Takamatsu D, Osaki M, Sekizaki T, Gottschalk M, Hamada S, Maruyama F. A locus encoding variable defence systems against invading DNA identified in Streptococcus suis. Genome Biol Evol. 2017; 9(4): 1000-1012.

78. Baig A, Weinert LA, Peters SE, Howell KJ, Chaudhuri RR, Wang J, Holden MT, Parkhill J, Langford PR, Rycroft AN, Wren BW, Tucker AW, Maskell DJ. Whole genome investigation of a divergent clade of the pathogen Streptococcus suis. Front Microbiol. 2015; 6: 1191. doi: 10.3389/fmicb.2015.01191.

79. Shi X, Ye H, Wang J, Li Z, Wang J, Chen B, Wen R, Hu Q, Feng Y. Loss of $89 \mathrm{~K}$ Pathogenicity Island in Epidemic Streptococcus suis, China. Emerg Infect Dis. 2016; 22(6): 1126-1127.

80. Wikipedia. Thomas Bayes. https://en.wikipedia.org/wiki/Thomas_ Bayes.

81. Wikipedia. Bayesian inference. https://en.wikipedia.org/wiki/Bayesianinference.

82. Du P, Zheng H, Zhou J, Lan R, Ye C, Jing H, Jin D, Cui Z, Bai X, Liang J, Liu J, Xu L, Zhang W, Chen C, Xu J. Detection of multiple parallel transmission outbreak of Streptococcus suis human infection by use of genome epidemiology, China, 2005. Emerg Infect Dis. 2017; 23 (2): 2014-2211.

83. Dawei G, Liping W, Chengping L. In vitro biofilms forming potential of Streptococcus suis isolated from human and swine in China. Braz J Microbiol. 2012; 43(3): 993-1004.

84. Bai J, Yang Y, Wang S, Gao L, Chen J, Ren Y, Ding W, Muhammad I, Li Y. Syringa iofil Lindl. Aqueous extract is a potential biofilms inhibitor in S. suis. Front Pharmacol. 2017; 8:26. doi: 10.3389/fphar.2017.00026

85. Bonifait L, Grignon L, Grenier D. Fibrinogen induces biofilms formation by Streptococcus suis and enhances its antibiotic resistance. Appl Environ Microbiol. 2008; 74(15): 4969-4972. 
86. Grenier D, Grignon L, Gottschalk M. Characterisation of biofilms formation by a Streptococcus suis meningitis isolate. Vet J. 2009; 179(2): 292-295.

87. Tanabe S, Bonifait L, Fittipaldi N, Grignon L, Gottschalk M, Grenier D. Pleiotropic effects of polysaccharide capsule loss on selected biological properties of Streptococcus suis. Can J Vet Res. 2010; 74(1): 65-70.

88. Wang Y, Zhang W, Wu Z, Lu C. Reduced virulence is an important characteristic of biofilms infection of Streptococcus suis. FEMS Microbiol Lett. 2011; 316(1): 36-43.

89. Meng X, Shi Y, Ji W, Meng X, Zhang J, Wang H, Lu C, Sun J, Yan Y. Application of a bacteriophage lysin to disrupt biofilms formed by the animal pathogen Streptococcus suis. Appl Environ Microbiol. 2011; 77(23): 8272-8279.

90. Wang S, Wang C, Gao L, Cai H, Zhou Y, Yang Y, Xu C, Ding W, Chen J, Muhammad I, Chen X, He X, Liu D, Li Y. Rutin inhibits Streptococcus suis biofilms formation by affecting CPS biosynthesis. Front. Pharmacol. 2017; 8:379. doi: 10.3389/fphar.2017.00379

91. Ding WY, Li YH, Lian H, Ai XY, Zhao YL, Yang YB, Han Q, Liu X, Chen $\mathrm{XY}, \mathrm{He} \mathrm{Z}$. Sub-minimum inhibitory concentrations of rhubarb water extracts inhibit Streptococcus suis biofilm formation. Front Pharmacol. 2017; 8:425. doi: 10.3389/fphar.2017.00425.
92. Wang Y, Yi L, Zhang Z, Fan H, Cheng X, Lu C. Biofilm formation host-cell adherence, and virulence genes regulation of Streptococcus suis in response to autoinducer-2 signaling. Curr Microbiol. 2014; 68(5): 575-580.

93. Wang Y, Yi L, Wang S, Fan H, Ding C, Mao X, Lu C. Crystal structure and identification of two key amino acids involved in AI-2 production and biofilm formation in Streptococcus suis LuxS. PLoS One. 2015; 10(10): e0138826. doi: 10.1371/journal.pone.0138826.

94. Ma F, Yi L, Yu N, Wang G, Ma Z, Lin H, Fan H. Streptococcus suis serotype 2 biofilms inhibit the formation of neutrophil extracellular traps. Front Cell Infect Microbiol. 2017; 7: 86. doi: 10.3389/fcimb.2017.00086.

95. Segura M. Streptococcus suis vaccines: candidate antigens and progress. Expert Rev Vaccines. 2015; 14(12): 1587-1608.

96. Jiang X, Yang Y, Zhu L, Gu Y, Shen H, Shan Y, Li X, Wu J, Fang W. Live Streptococcus suis type 5 strain XS0 45 provides cross-protection against infection by strains of types 2 and 9. Vaccine. 2016; 34(51): 6529-6538.

97. Wang J, Feng Y, Wang C, Zheng F, Hassan B, Zhi L, Li W, Yao Y, He E, Jiang S, Tang J. Genome-wide analysis of a avirulent and reveal the strain induces protective immunity against challenge with virulent Streptococcus suis Serotype 2. BMC Microbiol. 2017; 17(1): 67. doi: 10.1186/s12866-017-0971-0. 